H. Morikawa

Nagoya Math. J.

Vol. 148 (1997), 73-112

\title{
ON DIFFERENTIAL POLYNOMIALS, II
}

\author{
HISASI MORIKAWA
}

Abstract. In Part II, we shall be concerned with applications of classical invariant theory, to statistic physics and to theta functions. Main theorem in Chapter 2 is stated as follows:

For a partition function

$$
\xi(s)=\sum_{l=1}^{\infty} \gamma_{l} s^{d l}
$$

satisfying $\gamma_{l} \geq 0(l \geq 1)$ and $\alpha>0$, the $2 n$-apolar of $\xi(s)$

$$
A_{2 n}(\xi(s), \xi(s))=s^{2 n} \sum_{l=0}^{2 n}(-1)^{k}\left(\begin{array}{c}
2 n \\
k
\end{array}\right)\left(\frac{d}{d s}\right)^{2 n-k} \xi(s)\left(\frac{d}{d s}\right)^{k} \xi(s)
$$

has the expansion

$$
A_{2 n}\left(\xi(s), \xi(s)=\sum_{l=2}^{\infty} \beta_{n, l} s^{-\alpha l}\right.
$$

such that $\beta_{n, l} \geq 0(l \geq 2)$. This means, for a given partition function $\xi(s)$ with nonnegative relative probabilities, we construct a sequence of partition functions $A_{2 n}(\xi(s), \xi(s))_{n \geq 1}$ with the same properties, which may be considered a sequence of symbolical higher derivative of $\xi(s)$. The main theorem in Chapter 3 is stated as follows: For given theta functions $\varphi_{1}(z)$ and $\varphi_{2}(z)$ of level $n_{1}$ and $n_{2}$ respectively, in $g$ variables $z=\left(z_{1}, z_{2}, \ldots, z_{g}\right)$, then $r=\left(r_{1}, r_{2}, \ldots, r_{g}\right)$-apolar

$$
A_{r}\left(\varphi_{1}(z), \varphi_{2}(z)\right)=\sum_{0 \leq j \leq r-j} \frac{(-1)^{|j|}}{n_{1}^{|j|} n_{2}^{|r-j|}}\left(\begin{array}{l}
r \\
j
\end{array}\right)\left(\frac{\partial}{\partial z}\right)^{j} \varphi_{1}(z)\left(\frac{\partial}{\partial z}\right)^{r-j} \varphi_{2}(z)
$$

is a theta function of level $n_{1}+n_{2}$, and

$$
\begin{aligned}
\left(\frac{\partial}{\partial z}\right)^{h} \varphi_{1}(z)\left(\frac{\partial}{\partial z}\right)^{k} \varphi_{2}(z)= & \sum_{\substack{0 \leq j \leq h+k\\
}} \sum_{l}(-1)^{|l|} n_{1}^{|l|} n_{2}^{|\jmath-l|}\left(\begin{array}{c}
h \\
l
\end{array}\right)\left(\begin{array}{c}
k \\
j-l
\end{array}\right) \\
& \frac{(-1)^{|h|}\left(n_{1} n_{2}\right)^{|h+k-\jmath|}}{\left(n_{1}+n_{2}\right)^{|h+k|}}\left(\frac{\partial}{\partial z}\right)^{j} A_{h+k-\jmath}\left(\varphi_{1}(z), \varphi_{2}(z)\right) .
\end{aligned}
$$

Received October 2, 1995. 


\section{§2. Apolars of partition functions}

For a partition function $\zeta(s)$, we define its entropy $S(\zeta)$ and the central moments $M_{n}(\zeta)$ of $S(\zeta)$ as follows,

$$
\begin{aligned}
S(\zeta) & =-\frac{s \zeta^{(1)}(s)}{\zeta(s)}+\log \zeta(s) \\
M_{n}(\zeta) & =(-s)^{n} \sum_{l=0}^{n}(-1)^{l}\left(\begin{array}{l}
n \\
l
\end{array}\right) \frac{\zeta^{(n-l)}(s)}{\zeta(s)}\left(\frac{\zeta^{(1)}(s)}{\zeta(s)}\right)^{l} \quad(n \geq 2) .
\end{aligned}
$$

On the other hand, in the invariant theory on formal power series

$$
f(\xi \mid t)=\sum_{l=0}^{\infty}\left(\begin{array}{l}
\omega \\
l
\end{array}\right) \xi^{(l)} t^{l}
$$

the generators of semi-invariants are given by the same polynomial sequence,

$$
\phi_{n}(\xi)=\sum_{l=0}^{n}(-1)^{l}\left(\begin{array}{l}
n \\
l
\end{array}\right) \xi^{(n-l)} \xi^{(1) l} \xi^{(0) n-l-1} \quad(n \geq 2)
$$

i.e.,

$$
M_{n}(\xi)=\left(\frac{-s}{\xi(s)}\right)^{n} \phi_{n}(\xi) \quad(n \geq 2) .
$$

So we may define semi-invariants of partition functions. Most interesting semi-invariants of a partition function $\xi(s)$ are it's apolars,

$$
A_{2 n}(\xi, \xi)=s^{2 n} \sum_{l=0}^{2 n}(-1)^{l}\left(\begin{array}{c}
2 n \\
l
\end{array}\right) \xi^{(2 n-l)}(s) \xi^{(l)}(s) \quad(1 \leq n<\infty)
$$

For a partition function $\xi(s)=\sum_{l=1}^{\infty} \gamma_{l} s^{-\alpha l}$ with $\alpha>0$ and $\gamma_{l} \geq 0(l \geq 1)$, the $2 n$-apolar has the expansion

$$
A_{2 n}(\xi(s), \xi(s))=\sum_{l=0}^{\infty} \beta_{2 n, l} s^{-\alpha l}
$$

with non-negative coefficients

$$
\beta_{2 n, l}=\sum_{h=1}^{l-1} \gamma_{l-h} \gamma_{h} \sum_{j=0}^{2 n}(-1)^{j}\left(\begin{array}{c}
2 n \\
j
\end{array}\right)[\alpha h]^{(j)}[\alpha(l-h)]^{(2 n-j)},
$$


where

$$
\begin{aligned}
& {[\alpha h]^{(j)}[\alpha(l-h)]^{(2 n-j)}=\alpha(\alpha h+1)} \\
& \cdots(\alpha h+j-1)(\alpha(l-h))(\alpha(l-h)+1) \cdots(\alpha(l-h)+2 n-j+1) .
\end{aligned}
$$

MAIN THEOREM. Let $\xi(s)=\sum_{l=1}^{\infty} \gamma_{l}^{l=0} s^{-\alpha l}$ be a partition function, such that $\gamma_{l} \geq 0(l \geq 1)$ and $\alpha>0$. Then the $2 n$-apolar $A_{2 n}(\xi, \xi)$ has the expansion,

$$
\begin{aligned}
& \sum_{l=2}^{\infty} \beta_{2 n, l} s^{-\alpha l}, \\
& \beta_{2 n, l}=\sum_{h=1}^{l-1}\left\{\alpha^{2 n}(l-2 h)^{2 n}+c_{2 n, l, 1}(\alpha)(l-2 h)^{2(n-1)}+\right. \\
& \left.\quad \cdots+c_{2 n, l, n-1}(\alpha)(l-2 h)^{2}+c_{2 n, l, n}(\alpha)\right\} \gamma_{l-h} \gamma_{h} \quad(l \geq 2),
\end{aligned}
$$

with polynomials in $\alpha, c_{2 n, l, 1}(\alpha), \ldots, c_{2 n, l, n}(\alpha)$ whose coefficients are nonnegative integers. Consequently all the coefficients $\beta_{2 n, l}$ of the expansion of $A_{2 n}(\xi, \xi)$ are non-negative.

This suggests that from a partition functions $\zeta(s)$ with non-negative coefficients we may construct relating partition functions with non-negative coefficients, $A_{2}(\xi, \xi), A_{4}(\xi, \xi), A_{6}(\xi, \xi), \ldots$

\subsection{Outline of invariant theory on formal power series}

First of all let us recollect the outline of the invariant theory on formal power series

$$
f_{j}\left(\xi_{j} \mid t\right)=\sum_{l=0}^{\infty}\left(\begin{array}{c}
\omega_{j} \\
l
\end{array}\right) \xi_{j}^{(l)} t^{l} \quad(1 \leq j \leq r),
$$

where $\omega_{1}, \omega_{2}, \cdots, \omega_{r}$ are complex numbers and

$$
\xi_{j}=\left(\xi_{j}^{(0)}, \xi_{j}^{(1)}, \xi_{j}^{(2)}, \xi_{j}^{(3)}, \ldots\right) \quad(1 \leq j \leq r)
$$

are independent variable vectors of infinite length. The details of the theory are found in [3].

$s l(2, C)$-action on $\xi_{j}^{(0)}, \xi_{j}^{(1)}, \xi_{j}^{(2)}, \ldots$ is given by Cayley-Aronholdt operators,

$$
\left\{\begin{array}{l}
D \xi_{j}^{(l)}=l \xi_{j}^{(l-1)} \\
\Delta \xi_{j}^{(l)}=\left(\omega_{j}-l\right) \xi_{j}^{(l+1)} \\
H \xi_{j}^{(l)}=\left(\omega_{j}-2 l\right) \xi_{j}^{(l)} \quad(0 \leq l<\infty ; 1 \leq j \leq r),
\end{array}\right.
$$


with the relations,

$$
\left\{\begin{array}{l}
{[D, \Delta]=H} \\
{[H, D]=2 D} \\
{[H, \Delta]=-2 \Delta}
\end{array}\right.
$$

In $C\left[\xi_{1}, \xi_{2}, \ldots, \xi_{r}\right]$, degree, weight and index are defined as follows,

$$
\begin{array}{r}
\operatorname{deg} \xi_{j}^{(l)}=1, \quad \text { weight } \xi_{j}^{(l)}=l, \quad \operatorname{index} \xi_{j}^{(l)}=\omega_{j}-2 l \\
(0 \leq l<\infty ; 1 \leq j \leq r) .
\end{array}
$$

A polynomial $\varphi(\xi)$ in $C\left[\xi_{1}, \xi_{2}, \ldots, \xi_{r}\right]$ is called a semi-invariant of index $\nu$, if $\varphi(\xi)$ satisfies

$$
D \varphi(\xi)=0, \quad H \varphi(\xi)=\nu \varphi(\xi)
$$

Semi-invariants form a graded algebra

$$
S=\bigoplus_{\nu} S^{[\nu]}
$$

where $S^{[\nu]}$ is the vector space of semi-invariants of index $\nu$.

For a complex number $\nu$ we mean by $t^{\nu}$ the convergent power series

$$
\sum_{l=0}^{\infty}\left(\begin{array}{l}
\nu \\
l
\end{array}\right)(t-1)^{l}
$$

in the disc $\{t|| t-1 \mid<1\}$, which is a branch of the many valued function $t^{\nu}$.

The germ of $G L(2, C)$ acts on $f_{j}\left(\xi_{j} \mid t\right)(1 \leq j \leq r)$ as follows;

$$
f_{j}\left(\left(\begin{array}{c}
\delta \beta \\
\gamma \alpha
\end{array}\right) \xi_{j} \mid t\right)=(\gamma t+\delta)^{\omega_{j}} f_{j}\left(\xi_{j} \mid \frac{\alpha t+\beta}{\gamma t+\delta}\right) \quad(1 \leq j \leq r)
$$

i.e.,

$$
\left(\left(\begin{array}{c}
\delta \beta \\
\gamma \alpha
\end{array}\right) \xi_{j}\right)=\sum_{p=0}^{l} \sum_{q=0}^{\infty}\left(\begin{array}{l}
l \\
p
\end{array}\right)\left(\begin{array}{c}
\omega_{j}-l \\
q
\end{array}\right) \xi_{j}^{(l-p+q)} \alpha^{l-p} \beta^{q} \gamma^{p} \delta^{\omega_{j}-l-q}
$$




$$
\begin{aligned}
& \left(\left(\begin{array}{cc}
1 & 0 \\
\gamma & 1
\end{array}\right) \xi_{j}\right)^{(l)}=\sum_{p=0}^{l}\left(\begin{array}{l}
l \\
p
\end{array}\right) \xi^{(l-p)} \gamma^{p} \\
& \left(\left(\begin{array}{cc}
1 & \beta \\
0 & 1
\end{array}\right) \xi_{j}\right)^{(l)}=\sum_{q=0}^{\infty}\left(\begin{array}{c}
\omega_{j}-l \\
q
\end{array}\right) \xi_{j}^{(l-q)} \beta^{q} \\
& \left(\left(\begin{array}{cc}
\alpha^{-1} & 0 \\
0 & \alpha
\end{array}\right) \xi_{j}^{(l)}\right)=\alpha^{2 l-\omega_{j}} \xi_{j}^{(l)} \quad(0 \leq l<\infty ; 1 \leq j \leq r)
\end{aligned}
$$

A covariant of index $\nu$ means a formal power series

$$
F\left(\xi_{1}, \ldots, \xi_{r} ; t\right)=\sum_{l=0}^{\infty}\left(\begin{array}{l}
\nu \\
l
\end{array}\right) C_{l}(\xi) t^{l}
$$

with coefficients $C_{l}(\xi)$ in $C\left[\xi_{1}, \xi_{2}, \ldots, \xi_{r}\right]$ such that

$$
F\left(\left(\begin{array}{c}
\delta \beta \\
\gamma \alpha
\end{array}\right) \xi_{1}, \ldots,\left(\begin{array}{c}
\delta \beta \\
\gamma \alpha
\end{array}\right) \xi_{r} ; t\right)=(\gamma t+\delta)^{\nu} F\left(\xi_{1}, \ldots, \xi_{r} ; \frac{\alpha t+\beta}{\gamma t+\delta}\right)
$$

for the germ of $S L(2, C)$.

We denote by $C^{[\nu]}$ the vector space of covariants of index $\nu$, and we call the direct sum

$$
C=\oplus_{\nu} C^{[\nu]}
$$

the graded algebra of covariants.

The next four are the fundamental theorems in the invariant theory.

ThEOREM. (Robert's theorem) The mapping $\exp [t \Delta]$ defined by

$$
\exp [t \Delta] \varphi(\xi)=\sum_{l=0}^{\infty} \Delta^{l} \varphi(\xi) \frac{t^{(l)}}{l_{1}^{1}}
$$

is a graded algebra isomorphism of $S$ onto $C$ such that

$$
\exp [t \Delta] S^{[\nu]}=C^{[\nu]}
$$

TheOREM. (Structure theorem on the graded algebra of semi-invariants) Denoting polynomials

$$
\phi_{n}\left(\xi_{j}\right)=\sum_{l=0}^{\infty}(-1)^{l}\left(\begin{array}{l}
n \\
l
\end{array}\right) \xi_{j}^{(n-l)} \xi_{j}^{(1)^{l}} \xi_{j}^{(0)} \quad(2 \leq n<\infty ; 1 \leq j \leq r)
$$


we obtain a system of generators

$$
\begin{array}{cl}
\xi_{1}^{(0)}, \xi_{2}^{(0)}, \ldots, \xi_{r}^{(0)} ; \xi_{j}^{(0)} \xi_{k}^{(1)}-\xi_{k}^{(0)} \xi_{j}^{(1)} & (1 \leq i<k \leq r) \\
\phi_{n}\left(\xi_{j}\right) & (2 \leq n<\infty ; 1 \leq j \leq r)
\end{array}
$$

of the algebra $S\left[\xi_{1}^{(0)^{-1}}, \xi_{2}^{(0)^{-1}}, \ldots, \xi_{r}^{(0)^{-1}}\right]$.

Theorem. (Semi-simplicity theorem) Assume that

i) $\omega_{1}, \omega_{2}, \ldots, \omega_{r}$ are positive integers, or

ii) $\sum_{j=1}^{r} d_{j} \omega_{j} \neq 0,1,2,3, \ldots$ for all non-negative integral vector $\left(d_{1}\right.$, $\left.d_{2}, \cdots, d_{r}\right) \neq(0,0, \ldots, 0)$.

Then $C\left[\xi_{1}, \cdots, \xi_{r}\right]$ is a semi-simple sl $(2, C)$-module with decomposition

$$
C \oplus\left(\oplus_{\nu}\left(\operatorname{dim} S^{[\nu]}\right) W^{[\nu]}\right)
$$

where $W^{[\nu]}$ is the simple $s l(2, C)$-module with the basis $\left[e_{1}, e_{2}, e_{3}, \cdots\right]$ such that

$$
\begin{array}{ll}
\left(\begin{array}{ll}
0 & 0 \\
1 & 0
\end{array}\right) e_{l}=(\nu-l+1) e_{l-1}, & \left(\begin{array}{ll}
0 & 1 \\
0 & 0
\end{array}\right) e_{l}=(l+1) e_{l+1}, \\
\left(\begin{array}{cc}
-1 & 0 \\
0 & 1
\end{array}\right) e_{l}=(\nu-2 l) e_{l} . & \quad(0 \leq l \leq \infty)
\end{array}
$$

THEOREM. (Gram's theorem) Under the same condition on $\omega_{1}, \omega_{2}, \cdots$, $\omega_{r}$, the following two statements on an ideal I in $C\left[\xi_{1}, \xi_{2}, \ldots, \xi_{r}\right]$ are equivalent,

i) I is an $\operatorname{sl}(2, C)$-admissible ideal,

ii) there exists a set of index homogeneous semi-invariants $\left\{\varphi_{\lambda}\right\}_{\lambda \in \wedge}$ such that $I$ is generated by $\left\{\Delta^{\varrho} \varphi_{\lambda} \mid 0 \leq l<\infty, \lambda \in \wedge\right\}$.

\subsection{Central moments $M_{n}(Z)$ of entropy}

The scale-change invariant derivation

$$
\theta=-s \frac{d}{d s}
$$


is a nice tool for the study of partition functions.

We choose a generic partition function

$$
Z(s)=\exp [u(s)]
$$

and define the entropy of $Z(s)$ by

$$
S(Z)=\frac{\theta Z(s)}{Z(s)}+\log Z(s)=\theta u(s)+u(s)=(\theta+1) u(s) .
$$

The central $n$-th moment $M_{n}(Z)$ is defined by

$$
M_{n}(Z)=(-s)^{n} \sum_{l=0}^{n}\left(\begin{array}{l}
n \\
l
\end{array}\right) \frac{Z^{(n-l)}(s)}{Z(s)}\left(\frac{Z^{(1)}(s)}{Z(s)}\right)^{l} .
$$

Let us show the justification of the definitions in Boltzmann's sense. Let $\xi(s)$ be a partition function given by an integral

$$
\xi(s)=\int_{-\infty}^{\infty} \exp [-s f(x)] d x
$$

where we assume that $d / d x$ and the integral are freely commutative. Then we have

$$
\begin{aligned}
-\int_{-\infty}^{\infty} \log \left\{\frac{\exp [-s f(x)]}{\xi(s)}\right\} \frac{\exp [-s f(x)]}{\xi(s)} d x \\
=\int_{-\infty}^{\infty}(s f(x)+\log \xi(s)) \frac{\exp [-s f(x)]}{\xi(s)} d x \\
=\frac{-s \int_{-\infty}^{\infty} \frac{d}{d s} \exp [-s f(x)] d x}{\xi(s)}+\log \xi(s) \\
=\frac{-s \frac{d}{d s} \int_{-\infty}^{\infty} \exp [-s f(x)] d x}{\xi(s)}+\log \xi(s) \\
=-\frac{\frac{d}{d s} \xi(s)}{\xi(s)}+\log \xi(s)=\frac{\theta \xi(s)}{\xi(s)}+\log \xi(s)=(\theta+1) u(s) \\
\int_{-\infty}^{\infty}\left\{\log \left\{\frac{\exp [-s f(x)]}{\xi(s)}\right\}-S(Z)\right\}^{n} \frac{\exp [-s f(x)]}{\xi(s)} d x \\
=\int_{X}\left(-s f(x)+s \frac{\xi^{(1)}(s)}{\xi(s)}\right)^{n} \frac{\exp [-s f(x)]}{\xi(s)} d x
\end{aligned}
$$




$$
\begin{aligned}
& =(-s)^{n} \sum_{l=0}^{n}(-1)^{l}\left(\begin{array}{l}
n \\
l
\end{array}\right)\left(\frac{\xi^{(1)}(s)}{\xi(s)}\right)^{l} \frac{\int_{-\infty}^{\infty}\left(\frac{d}{d s}\right)^{n-l} \exp [-s f(x)] d x}{\xi(s)} \\
& =(-s)^{n} \sum_{l=0}^{n}(-1)^{l}\left(\begin{array}{l}
n \\
l
\end{array}\right)\left(\frac{\xi^{(1)}(s)}{\xi(s)}\right)^{l} \frac{\left(\frac{d}{d s}\right)^{n-l} \exp [-s f(x)] d x}{\xi(s)} \\
& =(-s)^{n} \sum_{l=0}^{n}(-1)^{l}\left(\begin{array}{l}
n \\
l
\end{array}\right) \frac{\xi^{(n-l)}(s)}{\xi(s)}\left(\frac{\xi^{(1)}(s)}{\xi(s)}\right)^{l} .
\end{aligned}
$$

LEMMA 2.1.

$$
\begin{aligned}
(-s)^{n}\left(\frac{d}{d s}\right)^{n} & =[\theta]^{(n)}=\theta(\theta+1) \cdots(\theta+n-1) \\
M_{2}(Z) & =\theta(\theta+1) u(s)=[\theta]^{(2)} u(s) \\
(-s)^{n}\left(\frac{d}{d s}\right)^{n} & =(\theta+2)(\theta+3) \cdots(\theta+n-1) M_{2}(Z) \\
& =[\theta+2]^{(n-2)} M_{2}(Z) .
\end{aligned}
$$

Proof. If we assume (2.19) for $n-1$,

$$
\begin{aligned}
(-s)^{n}\left(\frac{d}{d s}\right)^{n} & =\left(-s \frac{d}{d s}\right)(-s)^{n-1}\left(\frac{d}{d s}\right)^{n-1}+(n-1)(-s)^{n-1}\left(\frac{d}{d s}\right)^{n-1} \\
& =\theta[\theta]^{(n-1)}+(n-1)[\theta]^{(n-1)}(\theta+n-1)=[\theta]^{(n)}
\end{aligned}
$$

From the definition of $M_{2}(Z)$,

$$
\begin{aligned}
M_{2}(s) & =(-s)^{2}\left\{\frac{Z^{(1)}(s)}{Z(s)}-\left(\frac{Z^{(1)}(s)}{Z(s)}\right)^{2}\right\}=(-s)^{2} \frac{d}{d s}\left(\frac{\frac{d}{d s} Z(s)}{Z(s)}\right) \\
& =(-s)^{2}\left(\frac{d}{d s}\right)^{2} u(s)=\theta(\theta+1) u(s)=[\theta]^{(2)} u(s) .
\end{aligned}
$$

(2.21) is a direct consequence of (2.19) and (2.20).

Let us express the generating function

$$
M(Z, t)=1+\sum_{n=2}^{\infty} M_{n}(Z) \frac{t^{n}}{n !},
$$

where $M_{1}(Z) \equiv 0$. 
Proposition 2.1.

$$
\begin{aligned}
M(Z, t) & =\frac{Z(s(1-t))}{Z(s)} \exp \left[s t \frac{Z^{(1)}(s)}{Z(s)}\right] \\
M(Z, t) & =\exp \left[\sum_{j=2}^{\infty}(-s)^{j}\left(\frac{d}{d s}\right)^{j} u(s) \frac{t^{j}}{j !}\right] \\
& =\exp \left[\sum_{j=2}^{\infty}[\theta+2]^{(j-2)} M_{2}(Z) \frac{t^{j}}{j !}\right] .
\end{aligned}
$$

Proof. Using Tayler expansion, we have

$$
\begin{aligned}
& \frac{Z(s(1-t))}{Z(s)} \exp \left[s t \frac{Z^{(1)}(s)}{Z(s)}\right] \\
& =\left(\sum_{h=0}^{\infty} \frac{Z^{(h)}(s)}{Z(s)} \frac{(-s t)^{h}}{h !}\right)\left(\sum_{l=0}^{\infty}\left(\frac{Z^{(1)}(s)}{Z(s)}\right)^{l} \frac{(l t)^{l}}{l !}\right) \\
& =1+\sum_{n=2}^{\infty}\left\{\sum_{l=0}^{N}\left(\begin{array}{l}
n \\
l
\end{array}\right) \frac{Z^{(n-l)}(s)}{Z(s)}(-s t)^{n-l}\left(\frac{Z^{(1)}(s)}{Z(s)}\right)^{l}(s t)^{l}\right\} \\
& =1+\sum_{n=2}^{\infty}\left\{(-s)^{n} \sum_{l=0}^{n}(-1)^{l}\left(\begin{array}{l}
n \\
l
\end{array}\right) \frac{Z^{(n-l)}(s)}{Z(s)}\left(\frac{Z^{(1)}(s)}{Z(s)}\right)^{l}\right\} \frac{t^{n}}{n !} \\
& =1+\sum_{n=2}^{\infty} M_{n}(Z) \frac{t^{n}}{n !}=M(Z, t) \text {, } \\
& M(Z, t)=\frac{Z(s(1-t))}{Z(s)} \exp \left[s t \frac{Z^{(1)}(s)}{Z(s)}\right] \\
& =\exp \left[u(s-s t)-u(s)+s t \frac{d}{d s} u(s)\right] \\
& =\exp \left[\sum_{l=0}^{\infty}(-s)^{l}\left(\frac{d}{d s}\right)^{l} u(s) \frac{t^{l}}{l !}-u(s)-s \frac{d}{d s} u(s) t\right] \\
& =\exp \left[\sum_{j=2}^{\infty}(-s)^{j}\left(\frac{d}{d s}\right)^{j} u(s) \frac{t^{j}}{j !}\right]=\exp \left[\sum_{j=2}^{\infty}[\theta+2]^{(j-2)} M_{2}(Z) \frac{t^{j}}{j !}\right]
\end{aligned}
$$

We obtain the differential polynomial expressions $M_{n}(Z)$ in $M_{2}(Z)$ with respect to the derivation $\theta$. 
TheOREM 2.1 .

$$
\begin{aligned}
M_{n}(Z) & =n ! \sum_{\substack{\sum_{j} l_{j}=m, j \geq 2}} \prod_{j} \frac{1}{l_{j} !}\left(\frac{(-s)^{j}\left(\frac{d}{d s}\right)^{j} u(s)}{j !}\right)^{l_{j}} \\
& =n ! \sum_{\substack{\sum_{j} l_{j}=m, j \geq 2}} \prod_{j} \frac{1}{l_{j} !}\left(\frac{[\theta]^{(j-2)} M_{2}(Z)}{j !}\right)^{l_{j}} .
\end{aligned}
$$

Proof. From Proposition 2.1 it follows

$$
\begin{aligned}
& 1+\sum_{n=2}^{\infty} M_{n}(Z) \frac{t^{n}}{n !}=\exp \left[\sum_{j=2}^{\infty}(-s)^{j}\left(\frac{d}{d s}\right)^{j} u(s) \frac{t^{j}}{j !}\right] \\
& =\prod_{j=2}^{\infty} \exp \left[(-s)^{j}\left(\frac{d}{d s}\right)^{j} u(s) \frac{t^{j}}{j !}\right] \\
& =\prod_{j=2}^{\infty}\left\{\sum_{l_{j}=2}^{\infty}\left(\frac{(-s)^{j}\left(\frac{d}{d s}\right)^{j} u(s)}{j !}\right)^{l_{j}} \frac{t^{j l_{j}}}{l_{j} !}\right\} \\
& =1+\sum_{n=2}^{\infty}\left\{\sum_{\substack{\sum_{j} l_{j}=n, j \geq 2}} \prod_{j} \frac{1}{l_{j} !}\left(\frac{(-s)^{j}\left(\frac{d}{d s}\right)^{j} u(s)}{j !}\right)^{l_{j}} \frac{n}{!} t^{j l_{j}}\right\} \\
& =1+\sum_{n=2}^{\infty}\left\{n ! \sum_{\substack{\sum_{j} l_{j}=n, j \geq 2}} \prod_{j} \frac{1}{l_{j} !}\left(\frac{(-s)^{j}\left(\frac{d}{d s}\right)^{j} u(s)}{j !}\right)^{l_{j}}\right\} \frac{t_{n}}{n !} \\
& =1+\sum_{n=2}^{\infty}\left\{n ! \sum_{\substack{\sum_{j} l_{j}=n, j \geq 2}} \prod_{j} \frac{1}{l_{j} !}\left(\frac{[\theta+2]^{(j-2)} M_{2}(Z)}{j !}\right)^{l_{j}}\right\} \frac{t_{n}}{n !}
\end{aligned}
$$

$M(Z, s)$ satisfies a partial differential equation, from which we obtain a recurence relation between $\left(M_{n}(Z)\right)_{n \geq 2}$.

Proposition 2.2.

$$
s \frac{\partial}{\partial s} M(Z, t)+(1-t) \frac{\partial}{\partial t} M(Z, t)=t M_{2}(Z) M(Z, t)
$$


(2.29) $\quad \theta M_{n}(Z)=-s \frac{d}{d s} M_{n}(Z)$

$$
\begin{gathered}
=M_{n+1}(Z)-n M_{n}(Z)-n M_{2}(Z) M_{n-1}(Z) \quad(n \geq 2) . \\
(\theta+n) M_{n}(Z)=M_{n+1}(Z)-n M_{2}(Z) M_{n-1}(Z) \quad(n \geq 2) .
\end{gathered}
$$

Proof. From Proposition 2.1 it follows

$$
\begin{gathered}
M(Z, t)=\frac{Z(s(1-t))}{Z(s)} \exp \left[s t \frac{Z^{(1)}(s)}{Z(s)}\right] \\
=\frac{Z(s(1-t))}{Z(s)} \exp \left[s t \frac{d}{d s} u(s)\right],
\end{gathered}
$$

$$
\begin{aligned}
s \frac{\partial}{\partial s} M(Z, t)+(1-t) \frac{\partial}{\partial t} M(Z, t) & \\
= & \left(s \frac{\partial}{\partial s}+(1-t) \frac{\partial}{\partial t}\right) Z(s(1-t)) \frac{1}{Z(s)} \exp \left[s t \frac{d}{d s} u(s)\right] \\
& +\left(s \frac{\partial}{\partial s}+(1-t) \frac{\partial}{\partial t}\right)\left(\frac{1}{Z(s)}\right) Z(s(1-t)) \exp \left[s t \frac{d}{d s} u(s)\right] \\
& +\frac{Z(s(1-t))}{Z(s)}\left(s \frac{\partial}{\partial s}+(1-t) \frac{\partial}{\partial t}\right) \exp \left[s t \frac{d}{d s} u(s)\right] \\
= & -s \frac{d}{d s} u(s) Z(s(1-t)) \exp \left[s t \frac{d}{d s} u(s)\right] \\
& +(s t+s(1-t)) \frac{d}{d s} u(s) \frac{Z(s(1-t))}{Z(s)} \exp \left[s t \frac{d}{d s} u(s)\right] \\
& +\frac{Z(s(1-t))}{Z(s)} t s^{2}\left(\frac{d}{d s}\right)^{2} u(s) \exp \left[s t \frac{d}{d s} u(s)\right] \\
= & t(-s)^{2}\left(\frac{d}{d s}\right)^{2} \frac{Z(s(1-t))}{Z(s)} \exp \left[s t \frac{d}{d s} u(s)\right]=t M_{2}(Z) M(t)
\end{aligned}
$$

$$
\begin{aligned}
\left(s \frac{\partial}{\partial s}+\right. & \left.(1-t) \frac{\partial}{\partial t}\right) M(Z, t)-t M_{2}(Z) M(Z, t) \\
= & \sum_{n=2}^{\infty} \frac{d}{d s} M_{n}(Z) \frac{t^{n}}{n !}+(1-t) \sum_{n=2}^{\infty} M_{n}(Z) \frac{t^{n-1}}{(n-1) !} \\
& -t M_{2}(Z)-\sum_{n=2}^{\infty} M_{2}(Z) \frac{t^{n+1}}{n !}
\end{aligned}
$$

Comparing the coefficients of $t^{n}$ of the both sides, we obtain (2.28) and (2.29). 


\subsection{Inhomogeneous semi-invariants of partition functions}

For a given system of generic partition functions $\left(Z_{1}(s), Z_{2}(s), \ldots\right.$, $\left.Z_{r}(s)\right)$,

$$
\begin{aligned}
\xi_{j}^{(l)} \longrightarrow(-s)^{l} Z_{j}^{(l)}(s)=(-s)^{l} & \left(\frac{d}{d s}\right)^{l} Z_{j}(s) \\
& (0 \leq l<\infty ; 1 \leq j \leq r),
\end{aligned}
$$

so that

$$
\begin{aligned}
& D\left((-s)^{l} Z_{j}^{(l)}(s)\right)=l(-s)^{l-1} Z_{j}^{(l-1)}(s) \\
& \Delta\left((-s)^{l} Z_{j}^{(l)}(s)\right)=\left(\omega_{j}-l\right) Z_{j}^{(l+1)}(s) \quad(0 \leq l<\infty ; 1 \leq j \leq r) . \\
& H\left((-s)^{l} Z_{j}^{(l)}(s)\right)=\left(\omega_{j}-2 l\right) Z_{j}^{(l)}(s)
\end{aligned}
$$

The images of semi-invariants by the specialization are called semiinvariants of the system of partition functions $\left(Z_{1}(s), Z_{2}(s), \ldots, Z_{r}(s)\right)$. We denote by the same notations as $2, \Lambda$,

$$
S=\oplus_{\nu} S^{[\nu]}
$$

the graded algebra of semi-invariants of $\left(Z_{1}(s), Z_{2}(s), \cdots, Z_{r}(s)\right)$.

There are many standard ways to construct semi-invariants from given semi-invariants. One of most important one is making apolars.

Let $\varphi(s)$ and $\phi(s)$ be semi-invariants of index $\mu$ and $\nu$, respectively. Then $n$-th apolar of $\varphi(s)$ and $\phi(s)$ is defined by

$$
A_{n}(\varphi, \phi)(s)=\sum_{l=0}^{n}(-1)^{l}\left(\begin{array}{l}
n \\
l
\end{array}\right) \frac{\Delta^{n-l} \varphi(s)}{(\mu)_{n-l}} \frac{\Delta^{l} \phi(s)}{(\nu)_{l}} .
$$

The $n$-th apolar $A_{n}(\varphi, \phi)(s)$ is a semi-invariant of index $M-\nu-2 n$, which is independent on the choice of $\left(\omega_{1}, \omega_{2}, \ldots, \omega_{r}\right)$.

EXAMPLE.

$$
\begin{aligned}
& A_{n}\left(Z_{j}, Z_{k}\right)=\sum_{l=0}^{n}(-1)^{l}\left(\begin{array}{c}
n \\
l
\end{array}\right)(-s)^{n-l} Z_{j}^{(n-l)}(s)(-s)^{l} Z_{k}^{(l)}(s) \\
& A_{1}\left(Z_{j}, Z_{k}\right)=(-s) Z_{j}^{(1)}(s) Z_{k}(s)-Z_{j}(s)(-s) Z_{k}^{(1)}(s)
\end{aligned}
$$




$$
\begin{aligned}
A_{2}\left(Z_{j}, Z_{j}\right) & =2\left\{(-s)^{2} Z_{j}^{(2)}(s) Z_{j}(s)-\left((-s) Z_{j}^{(1)}(s)\right)^{2}\right\} \\
A_{2 m+1}\left(Z_{j}, Z_{j}\right) & =0 \\
A_{2 m}\left(Z_{j}, Z_{j}\right) & =\sum_{l=0}^{2 m}(-1)^{l}\left(\begin{array}{c}
2 m \\
l
\end{array}\right)(-s)^{2 m-l} Z_{j}^{(2 m-l)}(s)(-s)^{l} Z_{j}(s)
\end{aligned}
$$

Another standard way of construction is making Hankel determinants,

\section{$\operatorname{Hank}_{2 q}\left(Z_{j}\right)$}

$$
=\left|\begin{array}{ccccc}
Z_{j}(s), & (-s) Z_{j}^{(1)}(s), & (-s)^{2} Z_{j}^{(2)}(s), & \ldots, & (-s)^{q} Z^{(q)}(s) \\
(-s) Z_{j}^{(1)}(s), & (-s)^{2} Z_{j}^{(2)}(s), & (-s)^{3} Z_{j}^{(3)}(s), & \ldots,(-s)^{q+1} Z_{j}^{(q+1)}(s) \\
\vdots & \vdots & \vdots & \vdots \\
(-s)^{q} Z_{j}^{(q)}(s),(-s)^{q+1} Z_{j}^{(q+1)}(s),(-s)^{q+2} Z_{j}^{(q+2)}(s), \ldots, & (-s)^{2 q} Z_{j}^{(2 q)}(s)
\end{array}\right|
$$

EXAMPLE.

$$
\begin{aligned}
& \operatorname{Hank}_{2}\left(Z_{j}\right)=\left|\begin{array}{cc}
Z_{j}(s) & (-s) Z_{j}^{(1)}(s) \\
(-s) Z_{j}^{(1)}(s) & (-s)^{2} Z_{j}^{(2)}(s)
\end{array}\right| \\
& =(-s)^{2} Z_{j}^{(2)}(s) Z_{j}(s)-\left((-s) Z_{j}^{(1)}(s)\right)^{2}, \\
& \operatorname{Hank}_{4}\left(Z_{j}\right)=\left|\begin{array}{ccc}
Z_{j}(s) & (-s) Z_{j}^{(1)}(s) & (-s)^{2} Z^{(2)} \\
(-s) Z_{j}^{(1)}(s) & (-s)^{2} Z_{j}^{(2)}(s) & (-s)^{3} Z^{(3)}(s) \\
(-s)^{2} Z_{j}^{(2)}(s) & (-s)^{3} Z_{j}^{(3)}(s) & (-s)^{4} Z^{(4)}(s)
\end{array}\right| \\
& =(-s)^{4} Z_{j}^{(4)}(s)(-s)^{2} Z_{j}^{(2)}(s) Z_{j}(s) \\
& +2(-s)^{3} Z^{(3)}(s)(-s)^{2} Z_{j}^{(2)}(s)(-s) Z_{j}^{(1)}(s) \\
& -\left((-s)^{3} Z_{j}^{(3)}(s)\right)^{2} Z_{j}(s)-(-s)^{4} Z^{(4)}(s)\left((-s)^{2} Z_{j}^{(2)}(s)\right)^{2} \\
& -\left((-s)^{2} Z^{(2)}(s)\right)^{3}
\end{aligned}
$$

One more important construction is making Wronskians. Let $\varphi_{1}(Z), \varphi_{2}(Z)$, $\ldots, \varphi_{n}(Z)$ be semi-invariants of index $\nu_{1}, \nu_{2}, \ldots, \nu_{n}$, respectively. Wronskian of $\varphi_{1}(Z), \varphi_{2}(Z), \ldots, \varphi_{n}(Z)$ is defined by

$$
W_{n}\left(\varphi_{1}, \ldots, \varphi_{n}\right)(Z)
$$




$$
=\left|\begin{array}{cccc}
\varphi_{1}(Z) & \varphi_{2}(Z) & \cdots & \varphi_{n}(Z) \\
\frac{1}{\left(\nu_{1}\right)_{1}} \Delta \varphi_{1}(Z) & \frac{1}{\left(\nu_{2}\right)_{1}} \Delta \varphi_{2}(Z) & \cdots & \frac{1}{\left(\nu_{n}\right)_{1}} \Delta \varphi_{n}(Z) \\
\frac{1}{\left(\nu_{1}\right)_{2}} \Delta^{2} \varphi_{1}(Z) & \frac{1}{\left(\nu_{2}\right)_{2}} \Delta^{2} \varphi_{2}(Z) & \cdots & \frac{1}{\left(\nu_{n}\right)_{2}} \Delta^{2} \varphi_{n}(Z) \\
\vdots & \vdots & & \vdots \\
\frac{1}{\left(\nu_{1}\right)_{n-1}} \Delta^{n-1} \varphi_{1}(Z) & \frac{1}{\left(\nu_{2}\right)_{n-1}} \Delta^{n-1} \varphi_{2}(Z) & \cdots & \frac{1}{\left(\nu_{n}\right)_{n-1}} \Delta^{n-1} \varphi_{n-1}(Z)
\end{array}\right| .
$$

which is a semi-invariant of index $\sum_{j=1}^{n} \nu-n(n-1)$. Wronskians are independent on the choice of $\omega_{1}, \omega_{2}, \ldots, \omega_{n}$.

Since degree, weight and index are defined by

$$
\begin{aligned}
& \operatorname{deg}\left((-s)^{l} Z^{(l)}(s)\right)=1, \quad \text { weight }\left((-s)^{l} Z_{j}^{(l)}(s)=l,\right. \\
& \operatorname{index}\left((-s)^{l} Z^{(l)}(s)\right)=\omega_{j} \quad(0 \leq l<\infty ; 1 \leq j \leq r),
\end{aligned}
$$

from the index formula for semi-invariants, we observe

$$
S^{[\nu]}=\bigoplus_{\nu=\sum_{j} d_{\jmath} \omega_{\jmath}-2 p} S\left[d_{1}, d_{2}, \ldots, d_{r} ; p\right] .
$$

where $S\left[d_{1}, \ldots, d_{r} ; p\right]$ is the vector space of semi-invariants separately homogeneous of degree $\left(d_{1}, d_{2}, \ldots, d_{r}\right)$ an isobaric of weight $p$. The decomposition

$$
S=\bigoplus_{d_{1}, \ldots, d_{r}, p} S\left[d_{1}, d_{2}, \ldots, d_{r} ; p\right]
$$

is independent on the choice of $\omega_{1}, \omega_{2}, \ldots, \omega_{n}$.

DEFINITION 2.1. An inhomogeneous semi-invariant of homogeneous degree $\left(d_{1}, \ldots, d_{r}\right)$ and isobaric of weight $p$ is a polynomial $\varphi\left(\ldots,(-s)^{l} Z^{(l)}(s) /\right.$ $\left.Z_{j}(s), \ldots\right)$ in $(-s)^{l} Z_{j}^{(l)}(s)(0 \leq l<\infty ; 1 \leq j \leq r)$ such that $\varphi(\ldots$, $\left.(-s)^{l} Z^{(l)}(s), \ldots\right)$ belongs to $S\left[d_{1}, d_{2}, \ldots, d_{r} ; p\right]$.

THEOREM 2.2. The algebra of inhomogeneous semi-invariants $S$ is a polynomial algebra in

$$
\frac{(-s) Z_{j}^{(1)}(s)}{Z_{j}(s)}-\frac{(-s) Z_{1}^{(1)}(s)}{Z_{1}(s)} \quad(2 \leq j \leq r)
$$

and the central moments of entropies

$$
M_{l}\left(Z_{j}\right) \quad(2 \leq l<\infty ; 1 \leq j \leq r)
$$


i.e.

$$
S=C\left[\left(\frac{(-s) Z_{j}^{(1)}(s)}{Z_{j}(s)}-\frac{(-s) Z_{1}^{(1)}(s)}{Z(s)}\right)_{2 \leq j \leq r},\left(M_{l}\left(Z_{j}\right)\right)_{\substack{2 \leq l<\infty \\ 1 \leq j \leq r}}\right]
$$

This is a direct consequence of the structure theorem on semi-invariants, because

$$
\begin{aligned}
M_{l}\left(Z_{j}\right) & =\sum_{l=0}^{n}(-1)\left(\begin{array}{c}
n \\
l
\end{array}\right) \frac{(-s)^{n-l} Z_{j}^{n-l}(s)}{Z_{j}(s)}\left(\frac{(-s) Z_{j}^{(1)}(s)}{Z_{j}(s)}\right)^{l} \\
& =\frac{1}{Z_{j}(s)^{n}} \sum_{l=0}^{n}\left(\begin{array}{c}
n \\
l
\end{array}\right)(-s)^{n-l} Z^{(n-l)}(s)\left((-s) Z_{j}^{(1)}(s)\right)^{l} Z_{j}(s)^{n-l-1} \\
& \left.=\frac{1}{Z_{j}(s)^{n}} \phi_{n}(\xi)\right)\left.\right|_{\xi_{j}^{(l)}=(-s)^{l} Z_{j}^{(l)}(s)}
\end{aligned}
$$

\subsection{Apolars of realistic partition functions}

In the former paragraphes, we have treat only abstract formal aspects of partition functions and their semi-invariants, however the most essential points are reality and positivities of coefficients of expansions of partition functions and certain their semi-invariants.

In the present paragraph we mean by a realistic partion function, a partition function

$$
\xi(s)=\sum_{l=1}^{\infty} \gamma_{l} s^{-\alpha l}
$$

such that $\gamma_{l} \geq 0(l \geq 1)$ and $\alpha>0$.

In order to calculate the coefficients of $A_{2 n}(\xi, \xi)$, we need Stirling numbers $S_{l}^{(n)}$, which are defined by the coefficients in $[x]^{(n)}$,

$$
[x]^{(n)}=x(x+1) \cdots(x+n-1)=\sum_{l-1}^{n} S_{l}^{(n)} x^{l}
$$

LIST OF $S_{l}^{(n)}$.

$$
\begin{aligned}
& {[x]^{(1)}=x} \\
& {[x]^{(2)}=x^{2}+x} \\
& {[x]^{(3)}=x^{3}+3 x^{2}+2 x}
\end{aligned}
$$




$$
\begin{aligned}
& {[x]^{(4)} }=x^{4}+6 x^{3}+11 x^{2}+6 x \\
& {[x]^{(5)} }=x^{5}+10 x^{4}+35 x^{3}+50 x^{2}+24 x \\
& {[x]^{(6)} }=x^{6}+15 x^{5}+85 x^{4}+225 x^{3}+274 x^{2}+120 x \\
& {[x]^{(7)} }=x^{7}+21 x^{6}+175 x^{5}+735 x^{4}+1624 x^{3}+1764 x^{2}+720 x \\
& S_{l}^{(n)}(1 \leq l \leq n) \text { are calculated by the recurence relation: }
\end{aligned}
$$

$$
S_{n}^{(n)}=1, \quad S_{1}^{(n)}=(n-1) !, \quad S_{l}^{(n)}=S_{l-1}^{(n-1)}+(n-1) S^{(n-1)_{l}} .
$$

The coefficients of $2 n$-apolar of $\xi(s)=\sum_{l=1}^{\infty} \gamma_{l} s^{-\alpha l}$

$$
\begin{aligned}
A_{2 n}(\xi(s), \xi(s)) & =s^{2 n} \sum_{j=0}^{2 n}(-1)^{j}\left(\begin{array}{c}
2 n \\
j
\end{array}\right)\left(\frac{\partial}{\partial s}\right)^{(j)} \xi(s)\left(\frac{\partial}{\partial s}\right)^{2 n-j} \xi(s) \\
& =\sum_{l=2}^{\infty} \beta_{2 n, l} s^{-\alpha l}
\end{aligned}
$$

are given by

$$
\beta_{2 n, l}=\sum_{h=1}^{l-1} \gamma_{l-h} \gamma_{h} \sum_{j=0}^{2 n}(-1)^{j}\left(\begin{array}{c}
2 n \\
j
\end{array}\right)[\alpha h]^{(j)}[\alpha(l-h)]^{(2 n-j)} .
$$

The next inequalities are the key for the estimation of $\beta_{2 n, l}$.

THEOREM 2.3. For real-non-negative $x$ and $y$

$$
\sum_{j}(-1)^{j}\left(\begin{array}{c}
2 n \\
j
\end{array}\right)[x]^{(j)}[y]^{(2 n-j)} \geq 0
$$

$$
(y-x) \sum_{j}(-1)^{j}\left(\begin{array}{c}
2 n+1 \\
j
\end{array}\right)[x]^{(j)}[y]^{(2 n+1-j)} \geq 0 \quad(n=0,1,2, \cdots) .
$$

Proof. For $n=0,1$, we have

$$
\begin{gathered}
(y-x)\left(-[x]^{(1)}+[y]^{(1)}\right)=(y-x)^{2}>0 \\
{[x]^{(2)}-2[x]^{(1)}[y]^{(1)}+[y]^{(2)}=(x-y)^{2}+(x+y) \geq 0 .}
\end{gathered}
$$


Assuming (2.40) and (2.41) for $2 n$ and $2 n+1$, we have

$$
\begin{aligned}
& \sum_{j}(-1)^{j}\left(\begin{array}{c}
2 n+2 \\
j
\end{array}\right)[x]^{(j)}[y]^{(2 n+2-j)} \\
& =\sum_{j}(-1)^{j}\left(\begin{array}{c}
2 n+1 \\
j
\end{array}\right)[x]^{(j)}[y]^{(2 n+1-j+1)} \\
& +\sum_{j}(-1)^{j}\left(\begin{array}{c}
2 n+1 \\
j-1
\end{array}\right)[x]^{((j-1)+1)}[y]^{(2 n+1-(j-1))} \\
& =\sum_{j}(-1)^{j}\left(\begin{array}{c}
2 n+1 \\
j
\end{array}\right)[x]^{(j)}[y]^{(2 n+1-j)}(y+2 n+1-j) \\
& -\sum_{j}(-1)^{j-1}\left(\begin{array}{c}
2 n+1 \\
j-1
\end{array}\right)[x]^{(\jmath-1)}[y]^{(2 n+1-\jmath)}(x+j-1) \\
& =\sum_{j}(-1)^{j}\left(\begin{array}{c}
2 n+1 \\
j
\end{array}\right)[x]^{(j)}[y]^{(2 n+1-j)}(y+2 n+1-j-x-j) \\
& =\sum_{j}(-1)^{j}\left(\begin{array}{c}
2 n+1 \\
j
\end{array}\right)[x]^{(j)}[y]^{(2 n+1-j)}(y-x+2 n+1-j-j) \\
& =(y-x) \sum_{j}(-1)^{\jmath}\left(\begin{array}{c}
2 n+1 \\
j
\end{array}\right)[x]^{(j)}[y]^{(2 n+1-j)} \\
& +\sum_{j}(-1)^{j}\left(\begin{array}{c}
2 n+1 \\
j
\end{array}\right)(2 n+1-j)[x]^{(j)}[y]^{(2 n+1-j)} \\
& -\sum_{j}(-1)^{j}\left(\begin{array}{c}
2 n+1 \\
j
\end{array}\right) j[x]^{(j)}[y]^{(2 n+j-1)} \\
& \geq(2 n+1) \sum_{j}(-1)^{j}\left(\begin{array}{c}
2 n \\
j
\end{array}\right)[x]^{(j)}[y]^{(2 n-j+1)} \\
& +(2 n+1) \sum(-1)^{j-1}\left(\begin{array}{c}
2 n \\
j-1
\end{array}\right)[x]^{(j-1+1)}[y]^{(2 n-(j-1))} \\
& =(2 n+1) \sum_{j}(-1)^{j}\left(\begin{array}{c}
2 n \\
j
\end{array}\right)[x]^{(j)}[y]^{(2 n-j)}\{y+2 n-j+x+j\} \\
& =(2 n+1)(x+y+2 n) \sum_{j}(-1)^{j}\left(\begin{array}{c}
2 n \\
j
\end{array}\right)[x]^{(j)}[y]^{(2 n-j)} \geq 0,
\end{aligned}
$$




$$
\begin{aligned}
& (y-x) \sum_{j}(-1)^{j}\left(\begin{array}{c}
2 n+2+1 \\
j
\end{array}\right)[x]^{(j)}[y]^{(2 n+2-j+1)} \\
& =(y-x) \sum_{j}(-1)^{j}\left(\begin{array}{c}
2 n+2 \\
j
\end{array}\right)[x]^{j}[y]^{(2 n+2-j)}(y+2 n+2-j) \\
& +(y-x) \sum_{j}(-1)^{j}\left(\begin{array}{c}
2 n+2 \\
j-1
\end{array}\right)[x]^{(j-1)}(x+j-1)[y]^{(2 n+2-(j-1))} \\
& =(y-x) \sum_{j}(-1)^{j}\left(\begin{array}{c}
2 n+2 \\
j
\end{array}\right)[x]^{(j)}[y]^{(2 n+2-j)}(y+2 n+2-j-x-j) \\
& =(y-x) \sum_{j}(-1)^{j}\left(\begin{array}{c}
2 n+2 \\
j
\end{array}\right)[x]^{(j)}[y]^{(2 n+2-j)}(y-x+2 n+2-j-j) \\
& =(y-x)^{2} \sum_{j}(-1)^{j}\left(\begin{array}{c}
2 n+2 \\
j
\end{array}\right)[x]^{(j)}[y]^{(2 n+2-j)} \\
& +(y-x) \sum_{j}(-1)^{j}\left(\begin{array}{c}
2 n+2 \\
j
\end{array}\right)(2 n+2-j)[x]^{(j)}[y]^{(2 n+1-j)}(y+2 n+1-j) \\
& -(y-x) \sum_{j}(-1)^{j}\left(\begin{array}{c}
2 n+2 \\
j
\end{array}\right) j[x]^{(j-1)}(x+j-1)[y]^{(2 n+1-(j-1))} \\
& \geq(2 n+2)(y-x) \sum_{j}(-1)^{j}\left(\begin{array}{c}
2 n+1 \\
j
\end{array}\right)[x]^{(j)}[y]^{(2 n+1-j)}(y+2 n+1-j) \\
& +(2 n+2)(y-x) \sum_{j}(-1)^{j-1}\left(\begin{array}{c}
2 n+1 \\
j-1
\end{array}\right)[x]^{(j-1)}[y]^{(2 n+1-(j-1))}(x+j-1) \\
& =(2 n+2)(y-x) \sum_{j}(-1)^{j}\left(\begin{array}{c}
2 n+1 \\
j
\end{array}\right)[x]^{(j)}[y]^{(2 n+1-j)}(y+2 n+1-j+x+j) \\
& =(2 n+2)(x+y+2 n+1)(y-x) \sum_{j}(-1)^{j}\left(\begin{array}{c}
2 n+1 \\
j
\end{array}\right)[x]^{(j)}[y]^{(2 n+1-j)} \geq 0 .
\end{aligned}
$$

The main theorem is a direct consequence of the inequalities (2.40) and (2.41).

THEOREM 2.4. If $\alpha>0$ and $\gamma_{l} \geq 0(l \geq 0)$, the $2 n$-apolar $A_{2 n}(\xi(s)$, 
$\xi(s))$ of the realistic partition function

$$
\xi(s)=\sum_{l=1}^{\infty} \gamma_{l} s^{-\alpha l}
$$

has the expansion

$$
A_{2 n}(\xi(s), \xi(s))=\sum_{l=2}^{\infty} \beta_{2 n, l} s^{-\alpha l}
$$

with non-negative coefficients

$$
\beta_{2 n, l}=\sum_{h=1}^{l-1} \gamma_{l-h} \gamma_{h} \sum_{j=0}^{2 n}(-1)^{j}\left(\begin{array}{c}
2 n \\
j
\end{array}\right)[\alpha h]^{(j)}[\alpha(l-h)]^{(2 n-j)} \geq 0
$$

Proof. From the definition of $2 n$-apolar and from Theorem 2.3 it follows

$$
\begin{aligned}
A_{2 n}(\xi(s), \xi(s)) & \\
= & \sum_{h, k=1}^{\infty} \gamma_{h} \gamma_{k} A_{2 n}\left(s^{-\alpha h}, s^{-\alpha k}\right) \\
= & \sum_{k, h=1}^{\infty} \sum_{j=0}^{2 n}(-1)^{j}\left(\begin{array}{c}
2 n \\
j
\end{array}\right)(-\alpha h)\left(-\alpha h_{1}\right) \\
& \cdots(-\alpha h-j+1)(-\alpha k)(-\alpha k-1) \\
& \cdots(-\alpha k-2 n+j+1) s^{-\alpha(h+k)} \\
= & \sum_{l=2}^{\infty} \sum_{h=1}^{l-1} \gamma_{l-h} \gamma_{h}\left(\sum_{j=0}^{2 n}(-1)^{j}\left(\begin{array}{c}
2 n \\
j
\end{array}\right)[\alpha h]^{(j)}[\alpha(l-h)]^{(2 n-j)}\right) s^{-\alpha l} \\
\beta_{2 n, l}= & \sum_{h=1}^{l-1} \gamma_{l-h} \gamma_{h}\left(\sum_{j=1}^{2 n}(-1)^{j}\left(\begin{array}{c}
2 n \\
j
\end{array}\right)[\alpha h]^{(j)}[\alpha(l-h)]^{(2 n-j)}\right) \geq 0 .
\end{aligned}
$$

The next three are the concrete calculations of the coefficients of $A_{2}(\xi(s), \xi(s)), A_{4}(\xi(s), \xi(s))$ and $A_{6}(\xi(s), \xi(s))$.

EXAMPLE 2.1. Let $\xi(s)=\sum_{l=1}^{\infty} \gamma_{l} s^{-\alpha l}$ be a realistic partition function. Then we have the expansion,

$$
\begin{aligned}
A_{2}(\xi, \xi) & =s^{2}\left(2 \xi^{(2)}(s) \xi(s)-2 \xi^{(l)}(s)^{2}\right) \\
& =\sum_{l=2}^{\infty}\left[\sum_{h=1}^{l-1}\left\{\alpha^{2}(l-2 h)^{2}+\alpha l\right\} \gamma_{l-h} \gamma_{h}\right] s^{-\alpha l}
\end{aligned}
$$


Consequently $A_{2}(\xi, \xi)$ is also a realistic partition function.

Proof. Watching the list of Stirling numbers, we observe,

$$
\begin{aligned}
A_{2}(\xi, \xi)= & \sum_{l=2}^{\infty}\left[\sum _ { h = 1 } ^ { l - 1 } \left\{[\alpha h]^{(2)}+[\alpha(l-h)]^{(2)}\right.\right. \\
& \left.\left.-2[\alpha h]^{(1)}[\alpha(l-h)]^{(1)}\right\} \gamma_{l-h} \gamma_{h}\right] s^{-\alpha l} \\
= & \sum_{l=2}^{\infty}\left[\sum_{h=1}^{l-1}\left\{\alpha^{2}\left(l^{2}-4 l h+h^{2}\right)+\alpha l\right\} \gamma_{l-h} \gamma_{h}\right] s^{-\alpha l} \\
= & \sum_{h=1}^{\infty}\left[\sum_{h=1}^{l-1}\left\{\alpha^{2}(l-2 h)^{2}+\alpha l\right\} \gamma_{l-h} \gamma_{h}\right] s^{-\alpha l} .
\end{aligned}
$$

EXAMPLE 2.2. For a realistic $\xi(s)=\sum_{l=1}^{\infty} \gamma_{l} s^{-\alpha l}$, we have

$$
\begin{aligned}
A_{4}(\xi, \xi)= & s^{4}\left(2 \xi^{(4)}(s) \xi(s)-8 \xi^{(3)}(s) \xi^{(1)}(s)+6 \xi^{(2)}(s)^{2}\right) \\
= & \sum_{l=2}^{\infty}\left[\sum _ { h = 1 } ^ { l - 1 } \left\{\alpha^{4}(l-2 h)^{4}+\left(6 \alpha^{3} l+8 \alpha^{2}\right)(l-2 h)^{2}\right.\right. \\
& \left.\left.+3 \alpha^{2} l^{2}+6 \alpha l\right\} \gamma_{l-h} \gamma_{h}\right] s^{-\alpha l} .
\end{aligned}
$$

Consequently $A_{4}(\xi, \xi)$ is also a realistic partition function.

Proof. Watching the list of Stirling numbers, we observe,

$$
\begin{aligned}
A_{4}(\xi, \xi)= & \sum_{l=2}^{\infty}\left[\sum _ { h = 1 } ^ { l - 1 } \left\{[\alpha h]^{(4)}+[\alpha(l-h)]^{(4)}-4[\alpha h]^{(3)}[\alpha(l-h)]^{(1)}\right.\right. \\
& \left.\left.-4[\alpha(l-h)]^{(3)}[\alpha h]^{(1)}+6[\alpha h]^{(2)}[\alpha(l-h)]^{(2)}\right\} \gamma_{l-h} \gamma_{h}\right] s^{-\alpha l} \\
= & \sum_{l=2}^{\infty}\left[\sum _ { h = 1 } ^ { l - 1 } \left\{\alpha^{4} h^{4}+6 \alpha^{3} h^{3}+11 \alpha^{2} h^{2}+6 \alpha h+\alpha^{4}(l-h)^{4}\right.\right. \\
& +6 \alpha^{3}(l-h)^{3}+11 \alpha^{2}(l-h)^{2}+6 \alpha(l-h) \\
& -4\left(\alpha^{3} h^{3}+3 \alpha^{2} h^{2}+2 \alpha h\right)(\alpha(l-h)) \\
& -4\left(\alpha^{3}(l-h)^{3}+3 \alpha^{2}(l-h)+2 \alpha(l-h)\right) \alpha h \\
& \left.\left.+6\left(\alpha^{2} h^{2}+\alpha h\right)\left(\alpha^{2}(l-h)^{2}+\alpha(l-h)\right)\right\} \gamma_{l-h} \gamma_{h}\right] s^{-\alpha l}
\end{aligned}
$$




$$
\begin{aligned}
= & \sum_{l=2}^{\infty}\left[\sum _ { h = 1 } ^ { l - 1 } \left\{\alpha ^ { 4 } \left(h^{4}-4 h^{3}(l-h)+6 h^{2}(l-h)\right.\right.\right. \\
& \left.-4 h(l-h)^{3}+(l-h)^{4}\right)+6 \alpha^{3}\left(l^{3}-4 l^{2} h+4 l h^{2}\right) \\
& \left.\left.+\alpha^{2}\left(11 h^{2}-11(l-h)^{2}-10 h(l-h)\right)+6 \alpha l\right\} \gamma_{l-h} \gamma_{h}\right] s_{-\alpha l} \\
= & \sum_{l=2}^{\infty}\left[\sum _ { h = 1 } ^ { l - 1 } \left\{\alpha^{4}(l-2 h)^{4}+6 \alpha^{3} l(l-2 h)^{2}\right.\right. \\
& \left.\left.+8 \alpha^{2}(l-2 h)^{2}+3 \alpha^{2} l^{2}+6 \alpha l\right\} \gamma_{l-h} \gamma_{h}\right] s^{-\alpha l} \\
= & \sum_{l=2}^{\infty}\left[\sum _ { h = 1 } ^ { l - 1 } \left\{\alpha^{4}(l-2 h)^{4}+\left(6 \alpha^{3} l+8 \alpha^{2}\right)(l-2 h)^{2}\right.\right. \\
& \left.\left.+3 \alpha^{2} l^{2}+6 \alpha l\right\} \gamma_{l-h} \gamma_{h}\right] s^{-\alpha l} .
\end{aligned}
$$

EXAMPLE 2.3. For a realistic $\xi(s)=\sum_{l=1}^{\infty} \gamma_{l} s^{-\alpha l}$, we have

$$
\begin{aligned}
A_{6}(\xi, \xi)= & s^{6}\left(2 \xi^{(6)}(s) \xi(s)-12 \xi^{(5)}(s) \xi^{(1)}(s)\right. \\
& \left.+30 \xi^{(4)}(s) \xi^{(2)}(s)-20 \xi^{(3)}(s)^{(2)}\right) \\
= & \sum_{l=2}^{\infty}\left[\sum _ { h = 1 } ^ { l - 1 } \left\{\alpha^{6}(l-2 h)^{6}+\left(15 \alpha^{5} l+40 \alpha^{4}\right)(l-2 h)^{4}\right.\right. \\
& +\left(45 \alpha^{4} l^{2}+210 \alpha^{3} l+184 \alpha^{2}\right)(l-2 h)^{2} \\
& \left.\left.+15 \alpha^{3} l^{3}+90 \alpha^{2} l^{2}+120 \alpha l\right\} \gamma_{l-h} \gamma_{h}\right] s^{-\alpha l}
\end{aligned}
$$

Proof. Watching the list of Stirling numbers, we observe,

$$
\begin{aligned}
A_{6}(\xi, \xi)= & s^{6}\left(2 \xi^{(6)}(s) \xi(s)-12 \xi^{(5)}(s) \xi^{(1)}(s) 30 \xi^{(4)}(s) \xi^{(2)}(s)-20 \xi^{(3)}(s)^{2}\right) \\
= & \sum_{l=2}^{\infty}\left[\sum _ { h = 1 } ^ { l - 1 } \left\{[\alpha h]^{(6)}+[\alpha(l-h)]^{(6)}-6[\alpha h]^{(5)}[\alpha(l-h)]^{(1)}\right.\right. \\
& -6[\alpha(l-h)]^{(5)}[\alpha h]^{(1)}+15[\alpha h]^{(4)}[\alpha(l-h)]^{(2)} \\
& \left.\left.+15[\alpha(l-h)]^{(4)}[\alpha h]^{(2)}-20[\alpha h]^{(3)}[\alpha(l-h)]^{(3)}\right\} \gamma_{l-h} \gamma_{h}\right] s^{-\alpha l}
\end{aligned}
$$




$$
\begin{aligned}
& =\sum_{l=2}^{\infty}\left[\sum _ { h = 1 } ^ { l - 1 } \left\{\alpha^{6} h^{6}+15 \alpha^{5} h^{5}+85 \alpha^{4} h^{4}+225 \alpha^{3} h^{3}+274 \alpha^{2} h^{2}\right.\right. \\
& +120 \alpha h+\alpha^{6}(l-h)^{6}+15 \alpha^{5}(l-h)^{5}+85 \alpha^{4}(l-h)^{4} \\
& +225 \alpha^{3}(l-h)^{3}+274 \alpha^{2}(l-h)^{2}+120 \alpha(l-h) \\
& -6\left(\alpha^{5} h^{5}+10 \alpha^{4} h^{4}+35 \alpha^{3} h^{3}+50 \alpha^{2} h^{2}+24 \alpha h\right) \alpha(l-h) \\
& -6\left(\alpha^{5}(l-h)^{5}+10 \alpha^{4}(l-h)^{4}+35 \alpha^{3}(l-h)^{3}+50 \alpha^{2}(l-h)^{2}\right. \\
& +24 \alpha(l-h)) \alpha h+15\left(\alpha^{4} h^{4}+6 \alpha^{3} h^{3}+11 \alpha^{2} h^{2}\right. \\
& +6 \alpha h)\left(\alpha^{2}(l-h)^{2}+\alpha(l-h)\right)+15\left(\alpha^{4}(l-h)^{4}+6 \alpha^{3}(l-h)^{3}\right. \\
& \left.+11 \alpha^{2}(l-h)^{2}+6 \alpha(l-h)\right)\left(\alpha^{2} h^{2}+\alpha h\right) \\
& -20\left(\alpha^{3} h^{3}+3 \alpha^{2} h^{2}+2 \alpha h\right)\left(\alpha^{3}(l-h)^{3}\right. \\
& \left.\left.\left.+3 \alpha^{2}(l-h)^{2}+2 \alpha(l-h)\right)\right\}\right] \\
& =\sum_{l=2}^{\infty}\left[\sum _ { h = 1 } ^ { l - 1 } \left\{\alpha ^ { 6 } \left(l^{6}-12 l^{5} h+60 l^{4} h^{4}-160 l^{3} h^{3}+240 l^{2} h^{4}\right.\right.\right. \\
& \left.-192 l h^{5}+64 h^{6}\right)+15 \alpha^{5}\left(l^{5}-8 l^{4} h+24 l^{3} h-32 l^{2} h^{3}+16 l h^{4}\right) \\
& +5 \alpha^{4}\left(17 l^{4}-100 l^{3} h+228 l^{2} h^{2}-256 l h^{3}+128 h^{4}\right) \\
& +\alpha^{3}\left(225 l^{3}-840 l^{2} h+840 l h^{2}\right) \\
& \left.\left.+\alpha^{2}\left(274 l^{2}-736 l h+736 h^{2}\right)+120 \alpha l\right\} \gamma_{l-h} \gamma_{h}\right] s^{-\alpha l} \\
& =\sum_{l=2}^{\infty}\left[\sum _ { h = 1 } ^ { l - 1 } \left\{\alpha^{6}(l-2 h)^{6}+15 \alpha^{5} l(l-2 h)^{4}+5 \alpha^{4}\left(8(l-2 h)^{4}\right.\right.\right. \\
& \left.+9 l^{2}(l-2 h)^{2}\right)+210 \alpha^{3} l(l-2 h)^{2}+15 \alpha^{3} l^{3}+184 \alpha^{2}(l-2 h)^{2} \\
& \left.\left.+90 \alpha^{2} l^{2}+120 \alpha l\right\} \gamma_{l-h} \gamma_{h}\right] s^{-\alpha l} \\
& =\sum_{l=2}^{\infty}\left[\sum _ { h = 1 } ^ { l - 1 } \left\{\alpha^{6}(l-2 h)^{6}+\left(15 \alpha^{5} l+40 \alpha^{4}\right)(l-2 h)^{4}\right.\right. \\
& +\left(45 \alpha^{4} l^{2}+210 \alpha^{3} l+184 \alpha^{2}\right)(l-2 h)^{2} \\
& \left.\left.+15 \alpha^{3} l^{3}+90 \alpha^{2} l^{2}+120 \alpha l\right\} \gamma_{l-h} \gamma_{h}\right] s^{-\alpha l} \text {. }
\end{aligned}
$$

Proposition 2.3. If two realistic partition functions $\xi(s)=\sum_{l=1}^{\infty}$ 
$\gamma_{l} s^{-\alpha l}$ and $\tilde{\xi}(s)=\sum_{l=1}^{\infty} \tilde{\gamma}_{l} s^{-\alpha l}$ satisfy $A_{2}(\xi, \xi)=A_{2}(\tilde{\xi}, \tilde{\xi})$, then $\xi(s)=\tilde{\xi}(s)$.

Proof. Putting $A_{2}(\xi, \xi)=\sum_{l=2}^{\infty} \beta_{l} s^{-\alpha l}$, we observe

$$
\beta_{l}=\sum_{h=1}^{l-1}\left\{\alpha^{2}(l-2 h)^{2}+\alpha l\right\} \gamma_{l-h} \gamma_{h},
$$

$$
2 \alpha \gamma_{1}^{2}=2 \alpha \tilde{\gamma}_{1}^{2}
$$

$2 \sum_{r=1}^{m}\left\{\alpha^{2}(2 m+1-2 r)^{2}+(2 m+1) \alpha\right\} \gamma_{2 m+1-r} \gamma_{r}$
$=2 \sum_{r=1}^{m}\{\alpha(2 m+1-2 r)+(2 m+1) \alpha\} \tilde{\gamma}_{2 m+l-r} \tilde{\gamma}_{r} \quad(m=0,1,2,3, \ldots)$,

$$
\begin{aligned}
& 2 \sum_{r=1}^{m-1}\left\{\alpha^{2}(2 m-2 r)^{2}+2 m \alpha\right\} \gamma_{2 m-r} \gamma_{r}+2 m \alpha \gamma_{m}^{2} \\
& =2 \sum_{r=1}^{m-1}\left\{\alpha^{2}(2 m-2 r)^{2}+2 m \alpha\right\} \tilde{\gamma}_{2 m-r} \tilde{\gamma}_{r}+2 m \alpha \tilde{\gamma}_{m}^{2} \quad(m=1,2,3, \ldots) .
\end{aligned}
$$

Since $\alpha>0$ and $\tilde{\gamma}_{l}, \gamma_{l} \geq 0(l \geq 1)$, we conclude $\tilde{\gamma}_{l}=\gamma_{l}(l \geq 1)$.

\section{REFERENCES}

[1] I. h. Grace and Young, The algebra of Invariants, Cambridge, 1903.

[2] I. Schur, Vorlesungen iiber Invariantentheoretic, Springer, 1968.

[3] H. Morikawa, Some analytic and geometric applications of invariant theoretic method, Nagoya Math. J., 80 (1980), 1-42. 


\section{$\S 3$. Differential polynomials in theta functions}

In the present chapter we shall be concerned with differential polynomials in theta functions. On the algebra of differential polynomials in theta functions, Heisenberg Lie algebra acts so nicely that the subalgebra of theta functions coincides with the subalgebra consisting of so called semi-invariants. This means the invariant theoretic point of view is a helpful method. By analogy of Hilbert operator we are able to calculate concrete expressions, and again apolars take important parts.

\section{Notations.}

$$
\begin{aligned}
\mathbf{Z}_{\geq 0} & =\{\text { non-nagative integer }\} \\
\mathbf{Z}_{\geq 0}^{g} & =\left\{j=\left(j_{1}, j_{2}, \ldots, j_{j}\right) \mid j_{i} \in \mathbf{Z}_{\geq 0} \quad(1 \leq j \leq g)\right\}, \\
|j| & =j_{1}+j_{2}+\cdots+j_{g} \\
j+\varepsilon_{i} & =\left(j_{1}, \ldots, j_{i-1}, j_{i}+1, j_{i+1}, \ldots, j_{g}\right) \\
j-\varepsilon_{i} & = \begin{cases}\left(j_{1}, \ldots, j_{i-1}, j_{i}-1, j_{i+1}, \ldots, j_{g}\right) & \left(j_{i} \geq 1\right) \\
0 & \left(j_{i}=0\right),\end{cases} \\
j ! & =j_{1} ! j_{2} ! \cdots j_{g} !,\left(\begin{array}{l}
j \\
h
\end{array}\right)=\frac{j !}{(j-h) ! h !}=\left(\begin{array}{l}
j_{1} \\
h_{1}
\end{array}\right)\left(\begin{array}{l}
j_{2} \\
h_{2}
\end{array}\right) \cdots\left(\begin{array}{l}
j_{g} \\
h_{g}
\end{array}\right),
\end{aligned}
$$

$z=\left(z_{1}, z_{2}, \ldots, z_{g}\right), u=\left(u_{1}, u_{2}, \ldots, u_{g}\right):$ systems of complex variables independent each other,

$\tau$ : a complex symmetric $g \times g$-matrix whose imaginary part is positive definite,

$$
\begin{aligned}
\left(u+l+\frac{a}{n}\right)= & \left(u_{1}+l_{1}+\frac{a_{1}}{n}\right)^{j_{1}}\left(u_{2}+l_{2}+\frac{a_{2}}{n}\right)^{j_{2}} \\
& \ldots\left(u_{g}+l_{g}+\frac{a_{g}}{n}\right)^{j_{g}}, \\
\left(2 \pi \eta \sqrt{-1} u+\frac{\partial}{\partial Z}\right)^{j}= & \left(2 \pi \eta \sqrt{-1} u_{1}+\frac{\partial}{\partial Z_{1}}\right)^{j_{1}}\left(2 \pi \eta \sqrt{-1} u_{2}+\frac{\partial}{\partial Z_{2}}\right)^{j_{2}} \\
& \ldots\left(2 \pi \eta \sqrt{-1} u_{g}+\frac{\partial}{\partial Z_{g}}\right)^{j_{g}}
\end{aligned}
$$




\subsection{Theta functions and auxiliary theta functions}

First of all we shall recollect some basic definitions and results in the previous article [3].

DEFINITION 3.1. A theta function of level $n(n \geq 1)$ means an entire function $f(z)=\left(z_{1}, z_{2}, \ldots, z_{g}\right)$ satisfying the difference equations.

$$
\begin{aligned}
& f(z+\hat{b}+b \tau) \\
& \quad=\exp \left[-\pi n \sqrt{-1}\left\{b \tau^{t} b+2 z^{t} b\right\} f(Z) \quad\left((\hat{b}, b) \in \mathbf{Z}^{g} \times \mathbf{Z}^{g}\right) .\right.
\end{aligned}
$$

Denoting of $\theta_{0}^{(n)}$ the vector space of theta functions of level $n$, we obtain the graded algebra of theta functions.

$$
\theta_{0}=\bigoplus_{n \geq 1} \theta_{0}^{(n)}
$$

Theta series

$$
\begin{aligned}
& \vartheta^{(n)}\left[\begin{array}{c}
\frac{a}{n} \\
0
\end{array}\right](\tau \mid z) \\
& \quad=\sum_{l \in \mathbf{Z}^{g}} \exp \left[\pi n \sqrt{-1}\left\{\left(l+\frac{a}{n}\right) \tau^{t}\left(l+\frac{a}{n}\right)+2 z^{t}\left(l+\frac{a}{n}\right)\right\}\right] \\
& \left(a \in \mathbf{Z}^{g} / n \mathbf{Z}^{g}\right),
\end{aligned}
$$

form a canonical basis of $\theta_{0}^{(n)}$, and consequently

$$
\operatorname{dim}_{\mathbf{C}} \theta_{0}^{(n)}=n^{g} .
$$

DEFINITION 3.2. An auxiliary theta of level $n$ means a function $\varphi(u, z)$ $=\left(u_{1}, u_{2}, \ldots, u_{g}, z_{1}, z_{2}, \ldots, z_{g}\right)$ such that

1. $\varphi(u, z)$ is a polynomial in $u=\left(u_{1}, u_{2}, \ldots, u_{g}\right)$ whose coefficients are entire functions in $z=\left(z_{1}, z_{2}, \ldots, z_{g}\right)$,

2. $\varphi(u+b, z+\hat{b}+b \tau)=\exp \left[-\pi n \sqrt{-1}\left\{b \tau^{t} b+2 z^{t} b\right\}\right] \varphi(u, z)\left((\hat{b}, b) \in \mathbf{Z}^{g} \times\right.$ $\left.\mathbf{Z}^{g}\right)$.

Denoting of $\theta^{(n)}$ the vector space of auxiliary theta functions of level $n$, we obtain the graded algebra of auxiliary theta functions,

$$
\theta^{(n)} \cap \theta_{0}=\theta_{0}^{(n)} \quad(n \geq 1) .
$$


Auxiliary theta series

$$
\begin{aligned}
& \vartheta_{j}^{(n)}\left[\begin{array}{c}
\frac{a}{n} \\
0
\end{array}\right](\tau \mid u, Z)=\left(2 \pi n \sqrt{-1} u+\frac{\partial}{\partial z}\right)^{j} \vartheta^{(n)}\left[\begin{array}{c}
\frac{a}{n} \\
0
\end{array}\right](\tau \mid Z) \\
&=(2 \pi n \sqrt{-1})^{|j|} \sum_{l \in \mathbf{Z}^{g}}\left(u+l+\frac{a}{n}\right)^{j} \\
& \exp \left[\pi n \sqrt{-1}\left\{\left(l+\frac{a}{n}\right) \tau^{t}\left(l+\frac{a}{n}\right)+2 z^{t}\left(l+\frac{a}{n}\right)\right\}\right] \\
&\left(j \in \mathbf{Z}_{\geq 0}^{g}, a \in \mathbf{Z}^{g} / n \mathbf{Z}^{g}\right)
\end{aligned}
$$

from a canonical basis of $\theta^{(n)}$. Denoting by $\theta_{j}^{(n)}$ the vector space spanned by

$$
\vartheta_{j}^{(n)}\left[\begin{array}{l}
\frac{a}{n} \\
0
\end{array}\right](\tau \mid u, z) \quad\left(a \in \mathbf{Z}^{g} / n \mathbf{Z}^{g}\right),
$$

we obtain a fine decomposition of $\theta$,

$$
\begin{gathered}
\theta=\bigoplus_{j \in \mathbf{Z}_{\geq 0}^{g}}, \bigoplus_{n \geq 1} \theta_{j}^{(n)}, \theta^{(n)}=\bigoplus_{j \in \mathbf{Z}_{\geq 0}^{g}} \theta_{j}^{(n)} . \\
\theta_{j}=\Delta^{j} \theta_{0}, \theta_{j}^{(n)}=\Delta^{j} \theta_{0}^{(n)}, \quad\left(j \in \mathbf{Z}_{\geq 0}^{g}, n \geq 1\right)
\end{gathered}
$$

and consequently

$$
\operatorname{dim}_{\mathbf{C}} \theta_{j}^{(n)}=n^{g} \quad\left(n \geq 1, j \in \mathbf{Z}_{\geq 0}^{g}\right) .
$$

\subsection{Action of Heisenberg Lie algebra}

We denote the projection operators

$$
\begin{aligned}
\sigma_{j}: \theta & \longrightarrow \theta_{j} \\
\sigma^{(n)}: & \rightarrow \theta^{(n)} \quad\left(j \in \mathbf{Z}_{\neq 0}^{g}, n \neq 1\right) \\
\sigma_{j}^{(n)}: & : \theta \longrightarrow \theta_{j}^{(n)}
\end{aligned}
$$

We introduce differential operators, derivations, acting on $\theta$ as follows,

$$
\begin{aligned}
\varepsilon & =\sum_{n \geq 1} n \sigma^{(n)} \\
D_{i} & =\sum_{n \geq 1} \frac{1}{2 \pi \sqrt{-1}} \frac{\partial}{\partial u_{i}} \sigma^{(n)} \quad(1 \leq i \leq g), \\
\Delta_{i} & =\sum_{n \geq 1}\left(2 \pi n \sqrt{-1} u_{i}+\frac{\partial}{\partial z_{i}} \sigma^{(n)}\right.
\end{aligned}
$$


then the action on the canonical basis are given by

$$
\begin{aligned}
& \varepsilon \vartheta_{j}^{(n)}\left[\begin{array}{l}
\frac{a}{n} \\
0
\end{array}\right](\tau \mid u, z)=n \vartheta_{j}^{(n)}\left[\begin{array}{c}
\frac{a}{n} \\
0
\end{array}\right](\tau \mid u, z) \\
& D_{i} \vartheta_{j}^{(n)}\left[\begin{array}{c}
\frac{a}{n} \\
0
\end{array}\right](\tau \mid u, z)=n j_{i} \vartheta_{j-\varepsilon_{\imath}}^{(n)}\left[\begin{array}{c}
\frac{a}{n} \\
0
\end{array}\right](\tau \mid u, z) \\
& \Delta_{i} \vartheta_{j}^{(n)}\left[\begin{array}{c}
\frac{a}{n} \\
0
\end{array}\right](\tau \mid u, z)=\vartheta_{j+\varepsilon_{i}}^{(n)}\left[\begin{array}{c}
\frac{a}{n} \\
0
\end{array}\right](\tau \mid u, z) \\
&\left(1 \leq i \leq g, j \in \mathbf{Z}_{\geq 0}^{g}, a \in \mathbf{Z}^{g} / n \mathbf{Z}^{g}, n \geq 1\right) .
\end{aligned}
$$

The operators $\varepsilon, D_{1}, D_{2}, \ldots, D_{g}, \Delta_{1}, \Delta_{2}, \ldots, \Delta_{g}$ satisfy the Heisenberg Lie algebra relations;

$$
\begin{aligned}
{\left[\varepsilon, D_{i}\right] } & =\left[\varepsilon, \Delta_{i}\right]=\left[D_{i}, D_{k}\right]=\left[\Delta_{i}, \Delta_{k}\right]=0 \\
{\left[D_{i}, \Delta_{k}\right] } & = \begin{cases}\varepsilon & (i=k) \\
0 & (i \neq k),\end{cases}
\end{aligned}
$$

Denoting

$$
D^{l}=D_{1}^{l_{1}} D_{2}^{l_{2}} \cdots D_{g}^{l_{g}}, \quad \Delta^{l}=\Delta_{1}^{l_{1}} \Delta_{2}^{l_{2}} \cdots \Delta_{g}^{l_{g}} \quad\left(l \in \mathbf{Z}_{\geq 0}^{g}\right)
$$

we have

$$
\begin{aligned}
\frac{1}{p ! n|p|} D^{p} \vartheta_{j}^{(n)}\left[\begin{array}{l}
\frac{a}{n} \\
0
\end{array}\right](\tau \mid u, z)= & \left(\begin{array}{l}
j \\
p
\end{array}\right) \vartheta_{j-p}^{(n)}\left[\begin{array}{c}
\frac{a}{n} \\
0
\end{array}\right](\tau \mid u, z) \\
\frac{1}{j ! n|j|} D^{j} \vartheta_{j}^{(n)}\left[\begin{array}{c}
\frac{a}{n} \\
0
\end{array}\right](\tau \mid u, z)= & \vartheta^{(n)}\left[\begin{array}{c}
\frac{a}{n} \\
0
\end{array}\right](\tau \mid u, z) \\
& \left(j, p \in \mathbf{Z}_{\geq 0}^{g}, a \in \mathbf{Z}^{g} / n \mathbf{Z}^{g}, n \geq 1\right) .
\end{aligned}
$$

The next lemma is the key for our purpose, which is the analogy of Hilbert operators in the classical invariant theory.

LEMMA 3.1. Projection operators $\sigma_{j}^{(n)} ; \theta \rightarrow \theta_{j}^{(n)}$ are given concretely as follows;

$$
\sigma_{j}^{(n)}=\sum_{p \in \mathbf{Z}_{\geq 0}^{g}} \frac{(-1)^{|p|}}{p ! j ! n|p| n^{|j|}} \Delta^{p+j} D^{p+j} \sigma^{(n)} \quad\left(j \in \mathbf{Z}_{\geq 0}^{g}, n \geq 1\right) .
$$


Proof. It is sufficient to prove

$$
\left(\sum_{p} \frac{(-1)^{|p|}}{p ! j ! n^{|p+j|}} \Delta^{p+j} D^{p+j}\right) \vartheta_{k}^{(n)}\left[\begin{array}{l}
\frac{a}{n} \\
0
\end{array}\right](\tau \mid u, z)= \begin{cases}\vartheta_{n}^{(n)}\left[\begin{array}{l}
\frac{a}{n} \\
0
\end{array}\right](\tau \mid u, z) & (k=j) \\
0 & (k \neq j) .\end{cases}
$$

From (3.12) and (3.14) it follows

$$
\begin{aligned}
& \left(\sum_{p} \frac{(-1)^{|p|}}{p ! j ! n^{|p+j|}} \Delta^{p+j} D^{p+j}\right) \vartheta_{k}^{(n)}\left[\begin{array}{l}
\frac{a}{n} \\
0
\end{array}\right](\tau \mid u, z) \\
& =\left(\sum_{p} \frac{(-1)^{|p|}}{p ! n^{|p|}} \Delta^{p+j} D^{p}\right)\left(\frac{1}{j ! n^{|j|}} D^{j} \vartheta_{k}^{(n)}\left[\begin{array}{l}
\frac{a}{n} \\
0
\end{array}\right](\tau \mid u, z)\right) \\
& =\left(\sum_{p}(-1)^{|p|} \Delta^{p+j} \frac{1}{p ! n|p|} D^{p}\right)\left(\begin{array}{l}
k \\
j
\end{array}\right) \vartheta_{k-j}^{(n)}\left[\begin{array}{l}
\frac{a}{n} \\
0
\end{array}\right](\tau \mid u, z) \\
& =\sum_{p}(-1)^{|p|} \Delta^{p+j}\left(\begin{array}{c}
k \\
j
\end{array}\right)\left(\begin{array}{c}
k-j \\
p
\end{array}\right) \vartheta_{k-j-p}^{(n)}\left[\begin{array}{c}
\frac{a}{n} \\
0
\end{array}\right](\tau \mid u, z) \\
& =\left(\sum_{p}(-1)^{|p|}\left(\begin{array}{c}
k-j \\
p
\end{array}\right)\left(\begin{array}{l}
k \\
j
\end{array}\right) \vartheta_{k}^{(n)}\left[\begin{array}{c}
\frac{a}{n} \\
0
\end{array}\right](\tau \mid u, z)\right. \\
& = \begin{cases}\vartheta_{k}^{(n)}\left[\begin{array}{l}
\frac{a}{n} \\
0
\end{array}\right](\tau \mid u, z) & (k=j) \\
0 & (k \neq j)\end{cases}
\end{aligned}
$$

Since $\theta=\oplus_{j \in \mathbf{Z}_{\geq 0}^{g}} \Delta^{j} \theta_{0}$, each auxiliary theta $\varphi(u, z)$ can be expressed uniquely

$$
\varphi(u, z)=\sum_{j \in \mathbf{Z}_{\geq 0}^{g}} \Delta^{j} \Psi_{j}(z)
$$

with theta functions $\Psi_{j}(z)$. We denote the linear operators by

$$
L_{j} \varphi(u, z)=\Psi_{j}(z) \quad\left(j \in \mathbf{Z}_{\geq 0}^{g}\right)
$$

i.e.,

$$
\varphi(u, z)=\sum_{j} \Delta_{j}^{j} \Psi_{j}(z)
$$

From Lemma 3.1 we observe that 
LEMMA 3.2.

$$
\left\{\begin{array}{l}
L_{0}=\sum_{n \geq 1} \sum_{p \in \mathbf{Z}_{\geq 0}^{g}} \frac{(-1)^{|p|}}{p ! n|p|} \Delta^{p} D^{p} \sigma^{(n)} \\
L_{j}=\sum_{n \geq 1} \sum_{p \in \mathbf{Z}_{\geq 0}^{g}} \frac{(-1)^{|p|}}{p ! j ! n^{|j+p|}} \Delta^{p} D^{p+j} \sigma^{(n)}
\end{array}\left(j \in \mathbf{Z}_{\geq 0}^{g}\right)\right.
$$

Proof. Since $\Delta^{j}$ induces a vector space isomorphisms of $\theta_{0}^{(n)}$ onto $\Delta \theta_{0}^{(n)}$ and $\sigma_{j}+\Delta^{j} L_{j}$, from Lemma 3.1 we conclude

$$
L_{j}=\sum_{n \geq 1} \sum_{p \in \mathbf{Z}_{\geq 0}^{g}} \frac{(-1)^{(n)}}{p ! j ! n^{|p+j|}} \Delta^{p} D^{p+j} \sigma^{(n)} .
$$

LEMMA 3.3.

$$
L_{0} \Delta_{i}=0 \quad(1 \leq i \leq g) .
$$

Proof. For any theta functions $\Psi_{l}(z)$

$$
L_{0}\left(\sum_{l} \Delta^{l} \Psi_{l}(z)\right)=\Psi_{l}(z),
$$

hence $L_{0} \Delta^{l} \Psi_{l}(z)=0\left(l \neq 0 \in \mathbf{Z}_{\geq 0}^{g}\right)$.

LEMMA 3.4. For theta functions $\varphi_{1}(z)$ and $\varphi_{2}(z)$,

$$
\begin{array}{r}
L_{0}\left(\Delta^{j} \varphi_{1}(z) \Delta^{k} \varphi_{2}(z)\right)=(-1)^{|j|} L_{0}\left(\varphi_{1}(z) \Delta^{j+k} \varphi_{2}(z)\right) \\
\left(j, k \in \mathbf{Z}_{\geq 0}^{g}\right) .
\end{array}
$$

Proof. First we shall show

$$
\Delta^{j} \varphi_{1}(z) \Delta^{k} \varphi_{2}(z)-(-1)^{|j|} \varphi_{1}(z) \Delta^{j+k} \varphi_{2}(z) \in \bigcup_{1 \leq j \leq g} \Delta_{i} \theta
$$

by induction on $|j|$. Assuming $(*)$ for $j(|j| \leq m)$, then

$$
\begin{array}{r}
\Delta^{j+\epsilon_{\imath}} \varphi_{1}(z) \Delta^{k} \varphi_{2}(z)=\Delta_{i}\left(\Delta^{j} \varphi_{1}(z) \Delta^{k} \varphi_{2}(z)\right)-\Delta^{j} \varphi_{1}(z) \Delta^{k+\epsilon_{i}} \varphi_{2}(z) \\
=(-1)^{\left|j+\epsilon_{i}\right|} \varphi_{1}(z) \Delta^{j+k+\epsilon_{\imath}}+\text { an element in } \bigcup_{1 \leq p \leq g} \Delta_{p} \theta
\end{array}
$$

Hence from Lemma 3.3 we obtain

$$
L_{o}\left(\Delta^{j} \varphi_{1}(z) \Delta^{k} \varphi_{2}(z)\right)=(-1)^{|j|} L_{0}\left(\varphi_{1}(z) \Delta^{j+k} \varphi_{2}(z)\right) .
$$




\subsection{Apolars of theta functions}

We shall define apolars of theta functions and give the concrete expression of the linear operators

$$
L_{j}: \theta \longrightarrow \theta_{0} \quad\left(j \in \mathbf{Z}_{\geq 0}^{g}\right) .
$$

Definition 3.3. For each $r$ in $\mathbf{Z}_{\geq 0}^{g}$ the $r$-th apolar of theta functions $\varphi_{1}(z)$ in $\theta_{0}^{\left(n_{1}\right)}$ and $\varphi_{2}(z)$ in $\theta_{0}^{\left(n_{2}\right)}$ is defined by

$$
A_{r}\left(\varphi_{1}(z), \varphi_{2}(z)\right)=\sum_{0 \leq j \leq r} \frac{(-1)^{|j|}}{n_{1}^{|j|} n_{2}^{|r-j|}}\left(\begin{array}{l}
r \\
j
\end{array}\right) \Delta^{j} \varphi_{1}(z) \Delta^{r-j} \varphi_{2}(z)
$$

LEMMA 3.5. For $\varphi_{1}(z)$ in $\theta_{1}^{(n)}$ and $\varphi_{2}(z)$ in $\theta_{0}^{\left(n_{2}\right)}$,

$$
A_{r}\left(\varphi_{1}(z), \varphi_{2}(z)\right) \in \theta_{0}^{\left(n_{1}+n_{2}\right)} .
$$

Proof. Obviously $A_{r}\left(\varphi_{1}(z), \varphi_{2}(z)\right)$ belongs to $\theta^{\left(n_{1}+n_{2}\right)}$. It is sufficient to show $D_{i} A_{r}\left(\varphi_{1}(z), \varphi_{2}(z)=0(1 \leq i \leq g)\right.$. By induction on $|j|$ we can prove

$$
D_{i} \Delta^{j} \sigma^{n}=\left(n j_{i} \Delta^{j-\epsilon_{i}}+\Delta^{n} D_{i}\right) \sigma^{n}
$$

hence

$$
\begin{aligned}
& D_{i} A_{r}\left(\varphi_{1}(z), \varphi_{2}(z)\right) \\
& =\sum_{j} \frac{(-1)^{|j|}}{n_{1}^{|j|} n_{2}^{|r-j|}}\left(\begin{array}{l}
r \\
j
\end{array}\right)\left\{\left(D_{i} \Delta^{j} \varphi_{1}(z)\right) \Delta^{r-j} \varphi_{2}(z)\right. \\
& \left.+\Delta^{j} \varphi_{1}(z)\left(D_{i} \Delta^{r-j} \varphi_{2}(z)\right)\right\} \\
& =\sum_{j} \frac{(-1)^{|j|}}{n_{1}^{|j|} n_{2}^{|r-j|}}\left(\begin{array}{l}
r \\
j
\end{array}\right)\left\{n_{1} j_{i} \Delta^{j-\epsilon_{\imath}} \varphi_{1}(z) \Delta^{r-j} \varphi_{2}(z)\right. \\
& \left.+n_{2}\left(r_{i}-j_{i}\right) \Delta^{j} \varphi_{1}(z) \Delta^{r-j-\epsilon_{2}} \varphi_{2}(z)\right\} \\
& =\sum_{j} \frac{(-1)^{\sum_{k \neq i} j_{k}}}{n_{1}^{\sum_{k \neq \imath} j_{k}} n_{2}^{\sum_{k \neq k}\left(r_{k}-j_{k}\right)}} \\
& \prod_{k \neq i}\left(\begin{array}{c}
r_{k} \\
j_{k}
\end{array}\right)\left\{\sum_{j_{i}}(-1)^{j^{2}}\left(\begin{array}{c}
r_{i} \\
j_{i}
\end{array}\right) \frac{j_{i}}{n_{1}^{j_{i}-1} n_{2}^{r_{i}-j_{2}}} \Delta^{j-\epsilon_{i}} \varphi_{1}(z) \Delta^{r-j} \varphi_{2}(z)\right. \\
& \left.+\sum_{j_{i}}(-1)^{j_{i}}\left(\begin{array}{c}
r_{i} \\
j_{i}
\end{array}\right) \frac{r_{i}-j_{i}}{n_{1}^{j_{i}} n_{2}^{r_{2}-f_{i}-1}} \Delta^{j} \varphi_{1}(z) \Delta^{r-j-\epsilon_{\imath}} \varphi_{2}(z)\right\}
\end{aligned}
$$


From

$$
\sum_{j}(-1)^{j_{\imath}}\left(\begin{array}{c}
r_{i} \\
j_{i}
\end{array}\right)+\sum_{j}(-1)^{j_{i}}\left(\begin{array}{c}
r_{i} \\
j_{i}
\end{array}\right)\left(r-\left(j_{i}-1\right)\right)=0
$$

we observe $D_{i} A_{r}\left(\varphi_{1}(z), \varphi_{2}(z)\right)=0(1 \leq i \leq g)$.

THEOREM 3.1. For theta functions $\varphi_{1}(z)$ in $\theta_{0}^{\left(n_{1}\right)}$ and $\varphi_{2}(z)$ in $\theta_{0}^{\left(n_{2}\right)}$

$$
\begin{array}{r}
L_{0}\left(\Delta^{j} \varphi_{1}(z) \Delta^{k} \varphi_{2}(z)\right) \\
=(-1)^{|j|}\left(\frac{n_{1} n_{2}}{n_{1}+n_{2}}\right)^{|j+k|} A_{j+k}\left(\varphi_{1}(z), \varphi_{2}(z)\right) \\
\left(j, k \in \mathbf{Z}_{\geq 0}^{g}\right) .
\end{array}
$$

Proof. From (3.19)

$$
L_{0}\left(\varphi_{1}(z) \Delta^{k} \varphi_{2}(z)\right)=(-1)^{|j|} L_{0}\left(\varphi_{1}(z) \Delta^{j+k} \varphi_{2}(z)\right),
$$

it is sufficient to prove

$$
L_{0}\left(\varphi_{1}(z) \Delta^{k} \varphi(z)\right)=\left(\frac{n_{1} n_{2}}{n_{1}+n_{2}}\right)^{k} A_{k}\left(\varphi_{1}(z), \varphi_{2}(z)\right) .
$$

since $D^{p} \varphi_{1}(z)=0$ for $p \neq 0$ and (3.14) it follows,

$$
\begin{aligned}
L_{0} & \left(\varphi_{1}(z) \Delta^{k} \varphi_{2}(z)=\sum_{p} \frac{(-1)^{|p|}}{p !\left(n_{1}+n_{2}\right)^{|p|}} \Delta^{p} D^{p} \varphi_{1}(z) \Delta^{k} \varphi_{2}(z)\right. \\
= & \sum_{p} \frac{(-1)^{|p|} n_{2}^{|p|}}{\left(n_{1}+n_{2}\right)} \Delta^{P} \varphi_{1}(z) \frac{D^{k} \Delta^{k} \varphi_{2}(z)}{p ! n_{2}^{|P|}} \\
= & \sum_{p} \frac{(-1)^{|p|} n_{2}^{|p|}}{\left(n_{1}+n_{2}\right)^{|p|}} \Delta^{p} \varphi_{1}(z)\left(\begin{array}{l}
k \\
p
\end{array}\right) \Delta^{k-p} \varphi_{2}(z) \\
= & \sum_{q \leq p} \frac{(-1)^{|p|} n^{|p|}}{\left(n_{1}+n_{2}\right)^{|p|}}\left(\begin{array}{l}
k \\
p
\end{array}\right)\left(\begin{array}{l}
p \\
q
\end{array}\right) \Delta^{q} \varphi_{1}(z) \Delta^{k-q} \varphi_{2}(z) \\
= & \sum_{q \leq p}(-1)^{|p-q|}\left(\begin{array}{l}
k-q \\
p-q
\end{array}\right)\left(\frac{n_{2}}{n_{1}+n_{2}}\right) \\
& \times \frac{n_{1}^{|q|} n_{2}^{|k|}}{\left(n_{1}+n_{2}\right)^{|q|}} \frac{(-1)^{|q|}}{n_{1}^{|q|} n_{2}^{|k-q|}}\left(\begin{array}{l}
k \\
q
\end{array}\right) \Delta^{q} \varphi_{1}(z) \Delta^{k-q} \varphi_{2}(z)
\end{aligned}
$$




$$
\begin{aligned}
= & \sum_{q}\left(\sum_{l}(-1)^{|l|}\left(\begin{array}{c}
k-q \\
l
\end{array}\right)\left(\frac{n_{2}}{n_{1}+n_{2}}\right)^{|l|}\right) \\
& \times \frac{n_{1}^{|q|} n_{2}^{|k|}}{\left(n_{1}+n_{2}\right)^{|q|}} \frac{(-1)^{|q|}}{n_{1}^{|q|} n_{2}^{|k-q|}}\left(\begin{array}{c}
k \\
q
\end{array}\right) \Delta^{q} \varphi_{1}(z) \Delta^{k-q} \varphi_{2}(z) \\
= & \sum_{q}\left(1-\frac{n_{2}}{n_{1}+n_{2}}\right)^{|k-q|} \frac{n_{1}^{|q|} n_{2}^{|k|}}{\left(n_{1}+n_{2}\right)^{|q|}} \frac{(-1)^{|q|}}{n_{1}^{|q|} n_{2}^{|l-q|}}\left(\begin{array}{c}
k \\
q
\end{array}\right) \Delta^{q} \varphi_{1}(z) \Delta^{k-q} \varphi_{2}(z) \\
= & \sum_{q}\left(\frac{n_{1}}{n_{1}+n_{2}}\right)^{|k-q|}\left(\frac{n_{1}}{n_{1}+n_{2}}\right)^{|q|} n_{2}^{|k|} \frac{(-1)^{|q|}}{n_{1}^{|q|} n_{2}^{|k-q|}}\left(\begin{array}{c}
k \\
q
\end{array}\right) \Delta^{q} \varphi_{1}(z) \Delta^{k-q} \varphi_{2}(z) \\
= & \left(\frac{n_{1} n_{2}}{n_{1}+n_{2}}\right)^{|k|} A_{k}\left(\varphi_{1}(z), \varphi_{2}(z)\right),
\end{aligned}
$$

THEOREM 3.2. For theta functions $\varphi_{1}(z)$ in $\theta_{0}^{\left(n_{1}\right)}$ and $\varphi_{2}(z)$ in $\theta_{0}^{\left(n_{2}\right)}$

$$
\begin{array}{r}
L_{j}\left(\Delta^{h} \varphi_{1}(z) \Delta^{k} \varphi(z)\right)=\sum_{l}(-1)^{|l|} n_{1}^{|l|} n_{2}^{|j-l|}\left(\begin{array}{c}
h \\
l
\end{array}\right)\left(\begin{array}{c}
k \\
j-l
\end{array}\right) \\
\frac{(-1)^{|h|}\left(n_{1} n_{2}\right)^{|h+k-j|}}{\left(n_{1}+n_{2}\right)^{|h+k|}} A_{h+k-j}\left(\varphi_{1}(z), \varphi_{2}(z)\right) \\
\left(j, h, k \in \mathbf{Z}_{\geq 0}^{g} ; n_{1}, n_{2} \geq 1\right) .
\end{array}
$$

Proof. From Lemma 3.2 the operator $L_{j} \sigma^{(n)}$ can be written as follows

$$
L_{j} \sigma^{(n)}=L_{0}\left(\frac{1}{j ! n^{|j|}} D^{j} \sigma^{(n)}\right)
$$

hence, using (3.14), we have

$$
\begin{aligned}
L_{j}\left(\Delta^{h} \varphi_{1}(z) \Delta^{k} \varphi_{2}(z)\right) & \\
& =\frac{1}{\left(n_{1}+n_{2}\right)^{|j|}} L_{0}\left(\frac{1}{j !} D^{j}\left(\Delta^{h} \varphi_{1}(z) \Delta^{k} \varphi_{2}(z)\right)\right. \\
& =\frac{1}{\left(n_{1}+n_{2}\right)^{|j|}} L_{0}\left(\sum_{l} \frac{n_{1}^{|l|}}{l ! n_{1}^{|l|}} D^{l} \Delta^{h} \varphi_{1} \frac{n_{2}^{|j-l|}}{(j-l) ! n_{2}^{|j-l|}} D^{j-l} \Delta^{k} \varphi_{2}(z)\right) \\
& =\frac{1}{\left(n_{1}+n_{2}\right)^{|j|}} L_{0}\left(\sum_{l} n_{1}^{|l|} n_{2}^{|j-l|}\left(\begin{array}{c}
h \\
l
\end{array}\right)\left(\begin{array}{c}
k \\
j-l
\end{array}\right) \Delta^{h-l} \varphi_{1}(z) \Delta^{k-j+l} \varphi_{2}(z)\right) \\
& =\frac{1}{\left(n_{1}+n_{2}\right)^{|j|}} \sum_{l} n_{1}^{|l|} n_{2}^{|j-l|}\left(\begin{array}{c}
h \\
l
\end{array}\right)\left(\begin{array}{c}
k \\
j-l
\end{array}\right) L_{0}\left(\Delta^{h-l} \varphi_{1}(z) \Delta^{k-j+l} \varphi_{2}(z)\right)
\end{aligned}
$$




$$
\begin{aligned}
= & \frac{1}{\left(n_{1}+n_{2}\right)^{|j|}} \sum_{l} n_{1}^{|l|} n_{2}^{|j-l|}\left(\begin{array}{c}
h \\
l
\end{array}\right)\left(\begin{array}{c}
k \\
j-l
\end{array}\right)(-1)^{|h-l|} \\
& \times\left(\frac{n_{1} n_{2}}{n_{1}+n_{2}}\right)^{|h+k-j|} A_{h+k-j}\left(\varphi_{1}(z), \varphi_{2}(z)\right) \\
= & \left(\sum_{l}(-1)^{|l|} n_{1}^{|l|} n_{2}^{|j-l|}\left(\begin{array}{c}
h \\
l
\end{array}\right)\left(\begin{array}{c}
k \\
j-l
\end{array}\right)\right) \\
& \times \frac{(-1)^{h}\left(n_{1} n_{2}\right)^{|h+k-j|}}{\left(n_{1}+n_{2}\right)^{|h+k|}} A_{h+k-j}\left(\varphi_{1}(z), \varphi_{2}(z)\right) .
\end{aligned}
$$

Finally we obtain the next decomposition formula.

\section{THEOREM 3.3 .}

$$
\begin{aligned}
& \Delta^{h} \varphi_{1}(z) \Delta^{k} \varphi_{2}(z) \\
& =\sum_{0 \leq j \leq h+k} \sum_{l}(-1)^{|l|} n_{1}^{|l|} n_{2}^{|j-l|}\left(\begin{array}{c}
h \\
l
\end{array}\right)\left(\begin{array}{c}
k \\
j-l
\end{array}\right) \\
& \quad \times \frac{(-1)^{|h|}\left(n_{1} n_{2}\right)^{|h+k-j|}}{\left(n_{1}+n_{2}\right)^{|h+k|}} \Delta^{j} A_{h+k-j}\left(\varphi_{1}(z), \varphi_{2}(z)\right) \\
& \left(\varphi_{1}(z) \in \theta_{0}^{\left(n_{1}\right)}, \varphi_{2}(z) \in \theta_{0}^{\left(n_{2}\right)} ; h, k \in \mathbf{Z}_{\geq 0}^{g}\right) .
\end{aligned}
$$

Specialization $u=\left(u_{1}, \ldots, u_{g}\right) \rightarrow 0=(0, \ldots, 0)$ induces a graded algebra isomorphism of $\theta=\oplus_{j \in \mathbf{Z}_{\neq 0}^{g}} \theta_{j}$ onto the graded algebra $D\left(\theta_{0}\right)$ of differential polynomials in theta functions such that

$$
\Delta^{j} \varphi(z) \longrightarrow\left(\frac{\partial}{\partial z}\right)^{j} \varphi(z) \quad\left(\varphi(z) \in \theta_{0}\right)
$$

Since the apolars $A_{r}\left(\varphi_{1}(z), \varphi_{2}(z)\right)$ of $\varphi_{1}(z)$ in $\theta_{0}^{\left(n_{1}\right)}$ and $\varphi_{2}(z)$ in $\theta_{0}^{\left(n_{2}\right)}$ belong to $\theta_{0}^{\left(n_{1}+n_{2}\right)}$, we may write

$$
\begin{aligned}
& A_{r}\left(\varphi_{1}(z), \varphi_{2}(z)\right) \\
& \quad=\sum_{0 \leq j \leq r} \frac{(-1)^{|j|}}{n_{1}^{|j|} n_{2}^{|r-j|}}\left(\begin{array}{l}
r \\
j
\end{array}\right)\left(\frac{\partial}{\partial z}\right)^{j} \varphi_{1}(z)\left(\frac{\partial}{\partial z}\right)^{r-j} \varphi_{2}(z) .
\end{aligned}
$$

The next is the translation of Theorem 3.3 by means of the specialization $\Delta^{j} \rightarrow(\partial / \partial z)^{j}\left(j \in \mathbf{Z}_{\geq 0}^{g}\right)$. 
THEOREM 3.4.

$$
\begin{aligned}
& \left(\frac{\partial}{\partial z}\right)^{h} \varphi_{1}(z)\left(\frac{\partial}{\partial z}\right)^{k} \varphi_{2}(z) \\
& =\sum_{0 \leq j \leq h+k} \sum_{l}(-1)^{l} n_{1}^{|l|} n_{2}^{|j-l|}\left(\begin{array}{c}
h \\
l
\end{array}\right)\left(\begin{array}{c}
k \\
j-l
\end{array}\right) \\
& \quad \times \frac{(-1)^{|h|}\left(n_{1} n_{2}\right)^{|h+k-j|}}{\left(n_{1}+n_{2}\right)^{|h+k|}}\left(\frac{\partial}{\partial z}\right)^{j} A_{h+k-j}\left(\varphi_{1}(z), \varphi_{2}(z)\right) \\
& \quad\left(\varphi_{1}(z) \in \theta_{0}^{\left(n_{1}\right)}, \varphi_{2}(z) \in \theta_{0}^{\left(n_{2}\right)} ; h, k \in \mathbf{Z}_{\geq 0}^{g}\right) .
\end{aligned}
$$

\subsection{A differential algebra expansion of the field of abelian functions}

We mean by $\mathbf{A}$ the abelian veriety defined by the period matrix $(I, \tau)$, and mean by $\mathbf{V}_{0}$ the affine $g$-bundle over $\mathbf{A}$ defined by the quotient

$$
\mathbf{V}_{0}=\mathbf{C}^{g} \times \mathbf{C}^{g} / \sim
$$

where $(u, Z) \sim(u+b, z+\hat{b}+b \tau)\left((\hat{b}, b) \in \mathbf{Z}^{g} \times \mathbf{Z}^{g}\right)$.

We donote by $\Psi_{0}$ the field of abelian functions on $\mathbf{A}$, i.e., the quotients $\varphi_{1}(z) / \varphi_{2}(z)$ of theta functions of the same level.

Definition 3.4. $\quad F_{j} \Psi$ means the vector space over $\Psi_{0}$ spanned by

$$
\frac{\Delta^{k} \varphi_{1}(z)}{\varphi_{2}(z)} \quad(0 \leq k \leq j),
$$

where $\varphi_{1}(z)$ and $\varphi_{2}(z)$ run over theta functions of the same level. Then $F_{0} \Psi=\Psi_{0}$ and

$$
\Psi=\bigcup_{j \in \mathbf{Z}_{\geq 0}^{g}} F_{j} \Psi
$$

is a filtration of an algebra. We call the elements of $\Psi$ auxiliary abelian functions.

THEOREM 3.5. The algebra $\Psi$ of auxiliary abelian functions in a polynomial algebra over $\Psi_{0}$,

$$
\Psi=\Psi_{0}\left[\Delta_{1} \vartheta\left[\begin{array}{l}
o \\
o
\end{array}\right](\tau \mid z), \ldots, \Delta_{g} \vartheta\left[\begin{array}{l}
o \\
o
\end{array}\right](\tau \mid z)\right],
$$

where $\vartheta\left[\begin{array}{l}o \\ o\end{array}\right](\tau \mid z)$ is the theta series of level one. 
Proof. We shall prove

$(*)$

$$
F_{r} \Psi \subset \Psi_{0}\left[\Delta_{1} \vartheta\left[\begin{array}{l}
o \\
o
\end{array}\right](\tau \mid z), \ldots, \Delta_{g} \vartheta\left[\begin{array}{l}
o \\
o
\end{array}\right](\tau \mid Z)\right]
$$

by induction on $|r|$. Assume $(*)$ for $j$ satisfying $|j| \neq r$. Then taking $h, k$ such that $h+k=r,|h+k|=r$, from 3.4 we have

$$
\begin{aligned}
\frac{\Delta^{h} \vartheta\left[\begin{array}{l}
o \\
o
\end{array}\right](\tau \mid Z) \Delta^{k} \vartheta\left[\begin{array}{l}
o \\
o
\end{array}\right](\tau \mid Z)}{\vartheta\left[\begin{array}{l}
o \\
o
\end{array}\right](\tau \mid Z)^{2}} & \sum_{0 \leq j \leq j+k=r} \sum_{l}(-1)^{|l|}\left(\begin{array}{l}
h \\
l
\end{array}\right)\left(\begin{array}{c}
k \\
j-l
\end{array}\right) \\
& \times \frac{(-1)^{|h|}}{2^{|r|}} \frac{\Delta^{j} A_{r-j}\left(\vartheta\left[\begin{array}{l}
o \\
o
\end{array}\right](\tau \mid Z), \vartheta\left[\begin{array}{l}
o \\
o
\end{array}\right](\tau \mid Z)\right)}{\vartheta\left[\begin{array}{l}
o \\
o
\end{array}\right](\tau \mid Z)^{2}} \\
\equiv & \frac{(-1)^{|h|}}{2^{|h|}} \frac{\Delta^{r} \vartheta\left[\begin{array}{l}
o \\
o
\end{array}\right](\tau \mid Z)^{2}}{\vartheta\left[\begin{array}{l}
o \\
o
\end{array}\right](\tau \mid Z)^{2}} \equiv \frac{(-1)^{|h|}}{2^{|r|-1}} \frac{\Delta^{r} \vartheta\left[\begin{array}{l}
o \\
o
\end{array}\right](\tau \mid Z)}{\vartheta\left[\begin{array}{l}
o \\
o
\end{array}\right](\tau \mid Z)} \bmod \bigcup_{j \supsetneqq r} F_{j} \Psi .
\end{aligned}
$$

This means

$$
\frac{\Delta^{r} \vartheta\left[\begin{array}{l}
o \\
o
\end{array}\right](\tau \mid z)}{\vartheta\left[\begin{array}{l}
o \\
o
\end{array}\right](\tau \mid z)} \in \Psi_{0}\left[\Delta_{1} \vartheta\left[\begin{array}{l}
o \\
o
\end{array}\right](\tau \mid z), \ldots, \Delta_{g} \vartheta\left[\begin{array}{l}
o \\
o
\end{array}\right](\tau \mid z)\right]
$$

Again from (3.24), for theta functions $\varphi_{1}(z), \varphi_{2}(z)$ in $\theta_{0}^{(n)}$ we have

$$
\frac{\Delta^{h} \varphi_{1}(z)}{\varphi_{2}(z)} \frac{\Delta^{k} \vartheta\left[\begin{array}{l}
o \\
o
\end{array}\right](\tau \mid z)}{\vartheta\left[\begin{array}{l}
o \\
o
\end{array}\right](\tau \mid z)}
$$




$$
\begin{aligned}
&= \sum_{0 \leq j \leq h+k=r} \sum_{l}(-1)^{|l|} n^{|l|}\left(\begin{array}{l}
h \\
l
\end{array}\right)\left(\begin{array}{c}
k \\
j-l
\end{array}\right) \frac{(-1)^{|l|} n^{|r-j|}}{(n+1)^{|r|}} \\
& \times \frac{\Delta^{j} A_{r-j}\left(\varphi_{1}(z) \vartheta\left[\begin{array}{l}
o \\
o
\end{array}\right](\tau \mid z)\right)}{\varphi_{2}(z) \vartheta\left[\begin{array}{l}
o \\
o
\end{array}\right](\tau \mid z)} \\
& \equiv \frac{n^{|h|}}{(n+1)^{|r|}} \frac{\Delta^{r}\left(\varphi(z) \vartheta\left[\begin{array}{l}
o \\
o
\end{array}\right](\tau \mid z)\right)}{\varphi_{2}(z) \vartheta\left[\begin{array}{l}
o \\
o
\end{array}\right](\tau \mid z)} \\
& \equiv \frac{n^{|h|}}{(n+1)^{|r|}} \frac{\Delta^{r} \varphi_{1}(z)}{\varphi_{2}(z)}+\frac{n^{|h|}}{(n+1)^{|r|}} \frac{\Delta^{r} \vartheta\left[\begin{array}{l}
o \\
o
\end{array}\right](\tau \mid z)}{\vartheta\left[\begin{array}{l}
o \\
o
\end{array}\right](\tau \mid z)} \bmod \underbrace{}_{j} \Psi . \\
& \bigcup j \mid \neq r
\end{aligned}
$$

Choosing $h, k$ such that $h+k=r,|h|,|k| \leq r$, we conclude

$$
\frac{\left.\Delta^{r} \varphi_{(} z\right)}{\varphi_{2}(z)} \in \Psi_{0}\left[\Delta_{1} \vartheta\left[\begin{array}{l}
o \\
o
\end{array}\right](\tau \mid z), \ldots, \Delta_{g} \vartheta\left[\begin{array}{l}
o \\
o
\end{array}\right](\tau \mid z)\right]
$$

The next is the translation of Theorem 3.5.

THEOREM 3.6. The polynomial algebra over $\Psi_{0}$

$$
\Psi_{0}\left[\frac{\frac{\partial}{\partial z_{1}} \vartheta\left[\begin{array}{l}
o \\
o
\end{array}\right](\tau \mid z)}{\vartheta\left[\begin{array}{l}
o \\
o
\end{array}\right](\tau \mid z)}, \ldots, \frac{\frac{\partial}{\partial z_{g}} \vartheta\left[\begin{array}{l}
o \\
o
\end{array}\right](\tau \mid z)}{\vartheta\left[\begin{array}{l}
o \\
o
\end{array}\right](\tau \mid z)}\right]
$$

coincides with the algebra over $\Psi_{0}$ spanned by

$$
\frac{\left(\frac{\partial}{\partial z}\right)^{j} \vartheta^{(n)}\left[\begin{array}{l}
\frac{a}{n} \\
o
\end{array}\right](\tau \mid z)}{\vartheta^{(n)}\left[\begin{array}{l}
o \\
o
\end{array}\right](\tau \mid z)} \quad\left(j \in \mathbf{Z}_{\geq 0}^{g} ; a, b \in \mathbf{Z}^{g} / n \mathbf{Z}^{g} ; n \geq 1\right) .
$$


Moreover derivations act on generators as follows,

$$
\begin{aligned}
& \left(3.31 \frac{\partial}{\partial z_{j}}\left(\frac{\frac{\partial}{\partial z_{j}} \vartheta\left[\begin{array}{l}
o \\
o
\end{array}\right](\tau \mid z)}{\vartheta\left[\begin{array}{l}
o \\
o
\end{array}\right](\tau \mid z)}\right)\right. \\
& =\frac{\frac{\partial}{\partial z_{j}} \vartheta\left[\begin{array}{l}
o \\
o
\end{array}\right](\tau \mid z) \frac{\partial}{\partial z_{j}} \vartheta\left[\begin{array}{l}
o \\
o
\end{array}\right](\tau \mid z)}{\vartheta\left[\begin{array}{l}
o \\
o
\end{array}\right](\tau \mid z)}-\frac{1}{2} \frac{A_{\epsilon_{i}+\epsilon_{j}}\left(\vartheta\left[\begin{array}{l}
o \\
o
\end{array}\right](\tau \mid z), \vartheta\left[\begin{array}{l}
o \\
o
\end{array}\right](\tau \mid z)\right)}{\vartheta\left[\begin{array}{l}
o \\
o
\end{array}\right](\tau \mid z)^{2}}
\end{aligned}
$$

where

$$
\begin{aligned}
A_{\epsilon_{\imath}+\epsilon_{j}} & \left(\vartheta\left[\begin{array}{l}
o \\
o
\end{array}\right](\tau \mid z), \vartheta\left[\begin{array}{l}
o \\
o
\end{array}\right](\tau \mid z)\right) \\
& =2 \frac{\partial^{2}}{\partial z_{i} \partial z_{j}} \vartheta\left[\begin{array}{l}
o \\
o
\end{array}\right](\tau \mid z)-2 \frac{\partial}{\partial z_{i}} \vartheta\left[\begin{array}{l}
o \\
o
\end{array}\right](\tau \mid z) \frac{\partial}{\partial z_{j}} \vartheta\left[\begin{array}{l}
o \\
o
\end{array}\right](\tau \mid z) .
\end{aligned}
$$

The derivations $\partial / \partial z_{1}, \ldots, \partial / \partial z_{g}$ map the field $\Psi_{0}$ into itself.

\subsection{Decomposition of differential polynomials}

We choose a system of independent variables

$$
s=\left(s_{1}, s_{2}, \ldots, s_{g}\right)
$$

and a system of dependent variables

$$
Z(s)=\left(Z_{1}(s), Z_{2}(s), \ldots, Z_{N}(s)\right)
$$

with positive integral valued weight

$$
\text { weight } Z_{k}(s)=n_{k} \quad(n \leq k \leq N)
$$

we regard $\left(Z_{k}^{(j)}(s)\right)$ also a system of dependent variables with respect to $s=\left(s_{1}, s_{2}, \ldots, s_{g}\right)$, where $Z_{k}^{(j)}=(\partial / \partial s)^{j} Z_{k}(s)$ and weight $Z_{k}^{(j)}(s)=n_{k}$ $\left(j \in \mathbf{Z}_{\geq 0}^{g}, 1 \leq k \leq N\right)$.

We introduce a system of derivatives of the polynomial algebra

$$
\Omega=Q\left[\ldots, Z_{k}^{(j)}, \ldots\right]
$$


as follows,

$$
\left\{\begin{aligned}
\varepsilon & =\sum_{n \geq 1} n \sigma_{(n)} \\
D_{i} & =\sum_{j \in \mathbf{Z}_{\geq 0}^{g}, 1 \leq k \leq N} n_{k} j_{i} Z_{k}^{\left(j-\epsilon_{2}\right)} \frac{\partial}{\partial Z_{k}^{(j)}} \quad(1 \leq i \leq g), \\
\Delta_{i} & =\frac{\partial}{\partial s_{1}}
\end{aligned}\right.
$$

where

$$
\sigma^{(n)}: \Omega \longrightarrow \Omega^{(n)}
$$

means the projection of $\Omega$ onto the vector subspace $\Omega^{(n)}$ consisting of elements of weight $n$ in $\Omega$. Then the derivations $\varepsilon, D_{1}, \ldots, D_{g}, \Delta_{1}, \ldots, \Delta_{1}, \ldots$, $\Delta_{g}$ satisfy Heisenberg Lie algebra relations:

$$
\left\{\begin{array}{l}
{\left[\varepsilon, D_{i}\right]=\left[D_{i}, D_{k}\right]=\left[\Delta_{i}, \Delta_{k}\right]=0} \\
{\left[D_{i}, \Delta_{k}\right]=\left\{\begin{array}{l}
(i=k) \\
(i \neq k) .
\end{array}\right.}
\end{array}\right.
$$

Definition 3.5. An element $\phi$ in $\Omega^{(n)}$ is called a semi-invariant of weight $n$, if $D_{i} \phi=0(1 \leq i \leq g)$. Semi-invariants of weight $n$ form a vector space $\Omega_{0}^{(n)}$ and the graded algebra

$$
\Omega_{0}=\bigoplus^{n \geq 1} \Omega_{0}^{(n)}
$$

is called the graded algebra of semi-invariants in $\Omega$.

LEMMA 3.6.

$$
\begin{gathered}
D_{i} \Delta^{p}=p_{i} \varepsilon \Delta^{p-\epsilon_{i}}+\Delta^{p} D_{i} \quad\left(p \in \mathbf{Z}_{\geq 0}^{g}\right) \\
\frac{D^{p} \Delta^{j} \varphi}{n^{|p|} p !}=\left(\begin{array}{l}
j \\
p
\end{array}\right) \Delta^{j-p} \varphi \quad\left(j, p \in \mathbf{Z}_{\geq 0}^{g}, \varphi \in \Omega_{0}^{(n)}\right) .
\end{gathered}
$$

Proof. Assuming (3.34) for $p_{i}$, we have

$$
\begin{aligned}
D_{i} \Delta^{p+\epsilon_{i}} & =D_{i} \Delta^{p} \Delta_{i}=\left(p_{i} \epsilon \Delta^{p-\epsilon_{i}}+\Delta^{p} D_{i}\right) \Delta_{i} \\
& =p_{i} \epsilon \Delta^{p}+\Delta^{p} \epsilon+\Delta^{p+\epsilon^{\imath}} D_{i}=\left(p_{i}+1\right) \epsilon \Delta^{p}+\Delta^{p+\epsilon_{\imath}} D_{i} .
\end{aligned}
$$


THEOREM 3.7. We denote

$$
L_{j}=\sum_{n \geq 1} \sum_{p \in \mathbf{Z}_{\geq 0}^{g}} \frac{(-1)^{|p|}}{p ! j ! n|p+\epsilon|} \Delta^{p} D^{p+j} \sigma^{(n)}
$$

then

$$
\begin{aligned}
D_{i} L_{i} & =0 \\
L_{j} \Delta^{k} \mid \Omega_{0} & =\left\{\begin{array}{cc}
\operatorname{id}_{\Omega_{0}} & (j=k) \\
0 & (j \neq k)
\end{array}\right. \\
\sum_{j \in \mathbf{Z}_{\geq 0}^{g}} \Delta^{j} L_{j} & =\operatorname{id}_{\Omega}
\end{aligned}
$$

Proof. From the relation

$$
\sum_{p}(-1)^{|p|}\left(\begin{array}{c}
m \\
p
\end{array}\right)= \begin{cases}1 & (m=0) \\
0 & (m \neq 0),\end{cases}
$$

it follows

$$
\begin{aligned}
\sum_{j} \Delta^{j} L_{j} & =\sum_{n \geq 1} \sum_{p} \frac{(-1)^{|p|}}{n^{|j+p|} p ! j !} \Delta^{i+p} D^{j+p} \sigma^{(n)} \\
& =\sum_{n \geq 1} \frac{1}{m ! n|m|}\left(\sum_{p}(-1)^{|p|}\left(\begin{array}{c}
m \\
p
\end{array}\right)\right) \Delta^{m} D^{m} \sigma^{(n)}-\sum_{n \geq 1} \sigma^{(n)}=\operatorname{id}_{\Omega}
\end{aligned}
$$

From (3.35), for each $\varphi$ in $\Omega_{0}^{(n)}$, we have

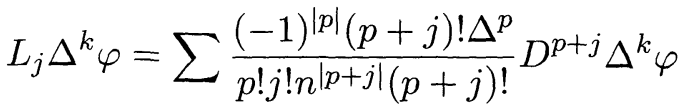

$$
\begin{aligned}
& =\sum(-1)^{|p|}\left(\begin{array}{c}
p+j \\
p
\end{array}\right)\left(\begin{array}{c}
k \\
p+j
\end{array}\right) \Delta^{p} \Delta^{k-j-p} \varphi \\
& =\sum_{p}(-1)^{|p|}\left(\begin{array}{c}
k-j \\
p
\end{array}\right)\left(\begin{array}{l}
k \\
j
\end{array}\right) \Delta^{k-j} \varphi=\varphi \text {. }
\end{aligned}
$$


This means $L_{j} \Delta^{k} \mid \Omega_{0}=\operatorname{id}_{\Omega_{0}}$. Since $\varepsilon \sigma^{(n)}=\eta \sigma^{(n)}$, from (3.34) we have

$$
\begin{aligned}
D_{i} L_{i}= & \sum_{n \geq 1} \sum_{p} \frac{(-1)^{|p|}}{n^{|p+j|} p ! j !} D_{i} \Delta^{p} D^{j+p} \sigma^{(n)} \\
= & \sum_{n \geq 1} \sum_{p} \frac{(-1)^{|p|}}{n^{|p+j|} p ! j !}\left(p+i \epsilon \Delta^{p_{\imath}-\epsilon_{i}} d j+p+\Delta^{p} D^{j+p+\epsilon_{\imath}}\right) \sigma^{(n)} \\
= & \sum_{n \geq 1}\left(\sum_{p} \frac{(-1)^{\left|p-\epsilon_{i}\right|}}{n^{\left|p-\epsilon_{\imath}+j\right|}\left(p-\epsilon_{i}\right) ! j !} \Delta^{p-1} D^{j+p}\right. \\
& \left.+\sum_{p} \frac{(-1)^{|p|}}{n^{|p+j|} p ! j !} \Delta^{p} D^{j+p+\epsilon_{i}}\right) \sigma^{(n)}=0
\end{aligned}
$$

Now we obtain the decomposition formula.

THEOREM 3.8. $L_{i}$ is a vector space isomorphism of $\Delta^{j} \Omega_{0}$ onto $\Omega_{0}$, and $\Delta^{j} L_{i}$ is the projection of $\Omega$ onto $\Delta^{j} \Omega_{0}$ in the decomposition:

$$
\Omega=\bigoplus_{j \in \mathbf{Z}_{\geq 0}^{g}} \Delta^{j} \Omega_{0}=\bigoplus_{n \geq 1} \bigoplus_{j \in \mathbf{Z}_{\geq 0}^{g}} \Delta^{j} \Omega_{0}^{(n)} .
$$

Theorem 3.8 states that each differential polynomial $\phi$ is uniquely written

$$
\phi=\sum_{h}\left(\frac{\partial}{\partial s}\right)^{h} L_{h} \phi
$$

with semi-invariant $L_{h} \phi$.

\section{REFERENCES}

[1] R. Hirota, A direct method of finding exact solution of non-linear evolution equations, Lecture Notes in Mathematics, No. 515 (1976), pp. 40-68.

[2] H. Morikawa, On Poisson brackets of semi-invariants, Manifolds and Lie groups, Progress in Math., Birkhäuser (1981), pp. 267-281.

[3] H. Morikawa, A decomposition theorem on differential Polynomials of theta functions, Nagoya Math. J., 96 (1984), 113-126.

The University of Aizu

Tsuruga, Ikki-machi, Aizu-Wakamatsu City

Fukushima, 965 Japan 